\title{
Evolución tectonotermal ordovícica del borde occidental del arco magmático Famatiniano: metamorfismo de las rocas máficas y ultramáficas de la Sierra de La Huerta- de Las Imanas (Sierras Pampeanas, Argentina)
}

\author{
Juan A. Murra \\ Edgardo G. Baldo
}

\author{
Departamento de Geología, Facultad de Ciencias Exactas, Físicas y Naturales, \\ Universidad Nacional de Córdoba y CONICET. \\ Av. Vélez Sarsfield 1611 (X5016GCA), Córdoba, Argentina. \\ jmurra@com.uncor.edu \\ ebaldo@com.uncor.edu
}

RESUMEN

\begin{abstract}
En el basamento pre Andino de las Sierras Pampeanas de Argentina, se registra una importante actividad magmática y tectonometamórfica comprendida entre el Ordovícico inferior y medio, vinculada al desarrollo y cierre de un arco magmático continental, que tuvo lugar durante la Orogenia Famatiniana como consecuencia de la aproximación de un supuesto terreno exótico (el Terreno Precordillera), que terminó anexándose al margen sur occidental del Gondwana (coordenadas actuales). En las sierras de La Huerta y Las Imanas, ubicadas en el límite occidental de este orógeno, afloran unidades ultramáficas (metaperidotitas con Ol-Opx-Cpx-Am-Spl y metapiroxenitas coroníticas con Opx-Cpx-SplAm-PI), máficas (metacuarzo-noritas con Opx-PI-Am-Qtz-Bt+Grt), e intermedias (metadioritas con Pl-Am-QtzBt-Ep), que son vinculadas al magmatismo del arco Famatiniano. Se reconocen además, rocas metasedimentarias (mármoles, gneises y migmatitas con Sil+Kfs+Grt) metamorfizadas en el Ordovícico Medio. Las rocas metaígneas máficas registran un primer metamorfismo (M1) de grado alto $\left(730^{\circ} \mathrm{C}\right.$ y $\left.8,4 \pm 0,5 \mathrm{kbar}\right)$, que es seguido de un segundo evento metamórfico de menor presión $\left(\mathrm{M} 2,720^{\circ} \mathrm{C}\right.$ y $\left.4,5 \mathrm{kbar}\right)$, registrado en la paragénesis Cum+Hbl+Mag dispuesta en coronas de reacción. Las rocas metaígneas ultramáficas preservan también la evidencia de dos etapas metamórficas (M1 y M2), pero solamente fue posible calcular las condiciones termobáricas del segundo evento, caracterizado por la formación de porfiroblastos de anfíboles. A la latitud de $32^{\circ} 30^{\prime} \mathrm{S}$, el arco magmático Famatiniano evidencia una zonación composicional transversal (en dirección este-oste), y que estaría relacionada con los actuales niveles de exposición. En este contexto, las unidades máficas y ultramáficas de las sierras de La Huerta y de Las Imanas representan los niveles más profundos del arco, lo cual es consistente con la mayor tasa de alzamiento y erosión propia de los bordes orogénicos.
\end{abstract}

Palabras claves:Orógeno Famatiniano, Sierras Pampeanas, Máfico-ultramáfico, Trayectoria PT, Argentina.

\section{ABSTRACT}

Tectonothermal ordovician evolution of the western margin of the Famatinian magmatic arc: metamorphism of the mafic and ultramafic rocks of Sierra de La Huerta -Las Imanas (Pampean Ranges, Argentina). An important magmatic and tectonometamorphic activity of Early and Middle Ordovician age is registered in the pre-Andean basement of the Sierras Pampeanas of Argentina. These were linked to the development of a continental magmatic arc during the Famatinian Orogeny, resulting from the approach and attachment of an alleged exotic terrane (the Precordillera Terrane), to the south western Gondwana's margin (present coordinates). A suit of meta-mafic 
and ultramafic rocks are exposed in the Sierras de La Huerta and Las Imanas, at the western limit of the Famatinian orogen. Metaperidotites (Ol-Opx-Cpx-Am-Spl), coronitic metapyroxenites (Opx-Cpx-Spl-Am-PI), metaquartz-norites (Opx$\mathrm{Pl}-\mathrm{Am}-\mathrm{Qtz}-\mathrm{Bt} \pm \mathrm{Grt}$ ) and metadiorites (PI-Am-Qtz-Bt-Ep) are associated with metasedimentary rocks (marbles, gneisses and migmatites with Sil+Kfs+Grt) that reached the peak and post-peak conditions of metamorphism at Middle Ordovician time. The meta-mafic rocks record a first high-grade metamorphic event (M1-730 ${ }^{\circ} \mathrm{C}$ and $8.4 \pm 0.5 \mathrm{kbar}$ ) and a second lower pressure event $\left(\mathrm{M} 2,720^{\circ} \mathrm{C}\right.$ and $\left.4.5 \mathrm{kbar}\right)$ with $\mathrm{Cum}+\mathrm{Hbl}+\mathrm{Mag}$ in a coronitic assemblage. The meta-ultramafic rocks also record the two metamorphic events, but only for the second one it was possible to calculate the P-T conditions. At latitude $32^{\circ} 30^{\prime} \mathrm{S}$, the Famatinian magmatic arc shows a systematic compositional variation normal to its trend, i.e. in an east-west direction, which could be related to present erosion levels. In this context, the mafic and ultramafic units of Sierras de La Huerta and Las Imanas, probably represent the deepest levels of the magmatic arc which is consistent with the position that they show marginal to the orogenic belt, i.e., where the uplift and erosion rates were larger.

Key words: Famatinian Orogen, Sierras Pampeanas, Mafic-ultramafic, PT-path, Argentina.

\section{INTRODUCCIÓN}

Las sierras de La Huerta y Las Imanas (SLH-SI) forman parte del conjunto de serranías que integran la provincia geológica de Sierras Pampeanas (SP) (Fig. 1). Esta gran unidad morfoestructural del basamento pre andino de Argentina está caracterizada por la presencia de rocas metamórficas del Proterozoicoy Paleozoico inferior, intruidas por granitoides cámbricos, ordovícicos, devónicos y carboníferos. Tanto el basamento metamórfico como las unidades plutónicas, han sido expuestos al actual nivel de erosión durante la tectónica compresional vinculada a la subducción de bajo ángulo de la Orogenia Andina (Jordan y Allmendinger, 1986). Las SP fueron divididas en dos subprovincias con características petrológicas y estructurales distintas: las Sierras Pampeanas Occidentales (SPOc), caracterizadas por la presencia de rocas del Proterozoico medio a superior, y las Sierras Pampeanas Orientales (SPOr), compuestas por rocas metamórficas del Cámbrico Inferior y la intrusión de grandes unidades batolíticas del Ordovícico Inferior a Medio y Devónico (Caminos, 1979; Ramos, 1999). Los dispersos afloramientos de las SPOc han sido integrados en un único terreno considerado alóctono al margen del Gondwana sur occidental (Terreno Precordillera, Astini et al., 1996; Astini y Thomas, 1999; o Terreno Cuyania, Ramos et al., 1996), en tanto que la formación de las unidades de las SPOr, se corresponden con la evolución de orógenos autóctonos a Gondwana (orógenos Pampeano, Famatiniano y Achaliano, fundamentalmente; Aceñolaza y Toselli, 1973; Rapela et al., 1998; Sims et al., 1998).

Las sierras de La Huerta y Las Imanas constituyen la continuación austral de la sierra de Valle Fértil (SVF).
Estas tres unidades serranas se ubican en la parte sureste de la provincia de San Juan, República Argentina (Figs. 1 y 2), y han sido consideradas originalmente como parte de las SPOc. Sin embargo, las investigaciones recientes permiten sugerir que las mismas corresponden en realidad al ámbito de las SPOr, ya que la edad de las rocas ígneas y metamórficas que las componen corresponde al Paleozoico Inferior (490-460 Ma; Pankhurst et al., 1998 y 2000; Rapela et al., 2001), siendo para esta época, parte del paleomargen del Gondwana sur occidental (coordenadas actuales; Baldo et al., 2001). Por otra parte, a la misma latitud los afloramientos de la sierra de Pie de Palo (Fig. 1) están formados por rocas mesoproterozoicas de 1000-1100 Ma (McDonough et al., 1993; Pankhurst y Rapela, 1998), y una cubierta neoproterozoica metamorfizada en el Ordovícico Medio (Casquet et al., 2001; Galindo et al., 2004a).

En las sierras de Las Imanas y de La Huerta, las unidades metamórficas son mayoritariamente derivadas de protolitos ígneos de carácter básico a intermedio (unidad metaígnea), y en menor porcentaje se observan rocas metamórficas derivadas de sedimentos pelíticos-samíticos (unidad metasedimentaria), y afloramientos esporádicos de rocas ultramáficas metamorfizadas, además de mármoles y anfibolitas (Vujovich etal., 1996; Castro de Machuca etal., 1996, 2004y 2005a; Murra, 2004 y bibliografías allí citadas). Los metasedimentos están intercalados con las rocas metaígneas máficas a intermedias y distribuidos de manera heterogénea a lo largo de toda la zona de estudio. Se reconocen, además, fajas discretas de cizallamiento dúctil de tipo inverso (Fig. 2), con ascen- 


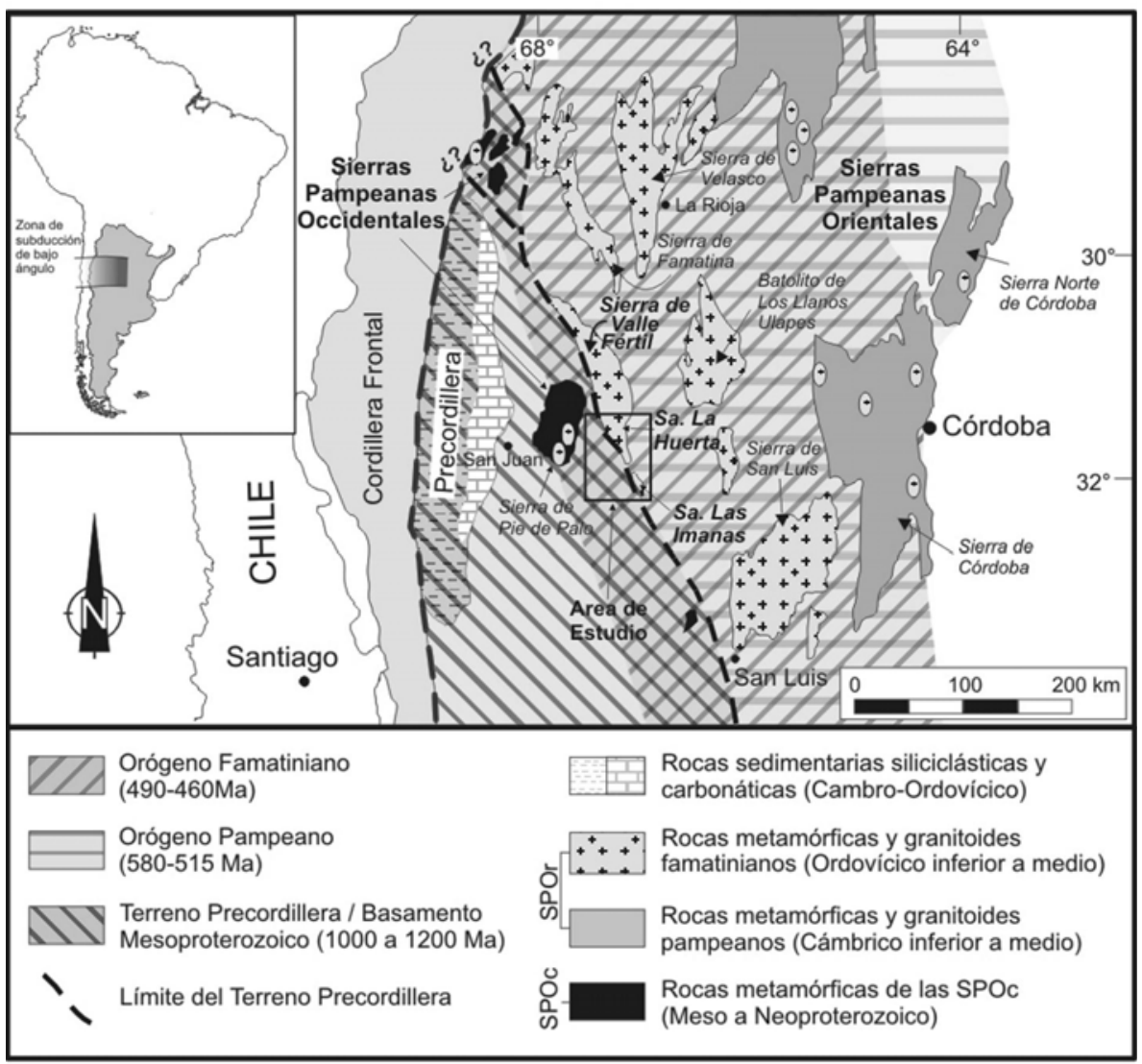

FIG. 1. Mapa geológico-geotectónico del sector centro-sur de las Sierras Pampeanas.

so del techo en dirección sureste (considerando la posición actual de los bloques), que retrabajan los protolitos metaígneos y metasedimentarios (Murra y Baldo, 2001; Murra, 2004; Castro de Machuca et al., 2004).

La información geoquímica, termobarométrica y geocronológica recopilada en el presente trabajo, permite reforzar el concepto del Cinturón Orogénico Famatiniano (Aceñolaza y Toselli, 1973) como una etapa concreta de evolución cortical dentro del ámbito de las Sierras Pampeanas. En general, los datos petrológicos concuerdan con el modelo de aproxima- ción de una placa litosférica (Terreno Precordillera) al margen sur-occidental de Gondwana (coordenadas actuales), generando la subducción hacia el este de la placa litosférica del alóctono, y la formación de un importante arco magmático continental, seguido de una etapa de colisión y posterior exhumación. Por otra parte, el estudio petrológico y termobarométrico de las rocas metaígneas de las sierras de La Huerta y Las Imanas, permite explicar la zonación magmáticametamórfica en sentido este-oeste del mencionado orógeno a esta latitud.

\section{SÍNTESIS GEOCRONOLÓGICA}

Las unidades ígneas y metamórficas aflorantes en las sierras de Las Imanas y de La Huerta se vinculan, temporal y espacialmente, con el evento tectono- metamórfico y magmático de la Orogenia Famatiniana, y son correlacionables con las unidades ubicadas inmediatamente al norte, en el área de la sierra de Valle 


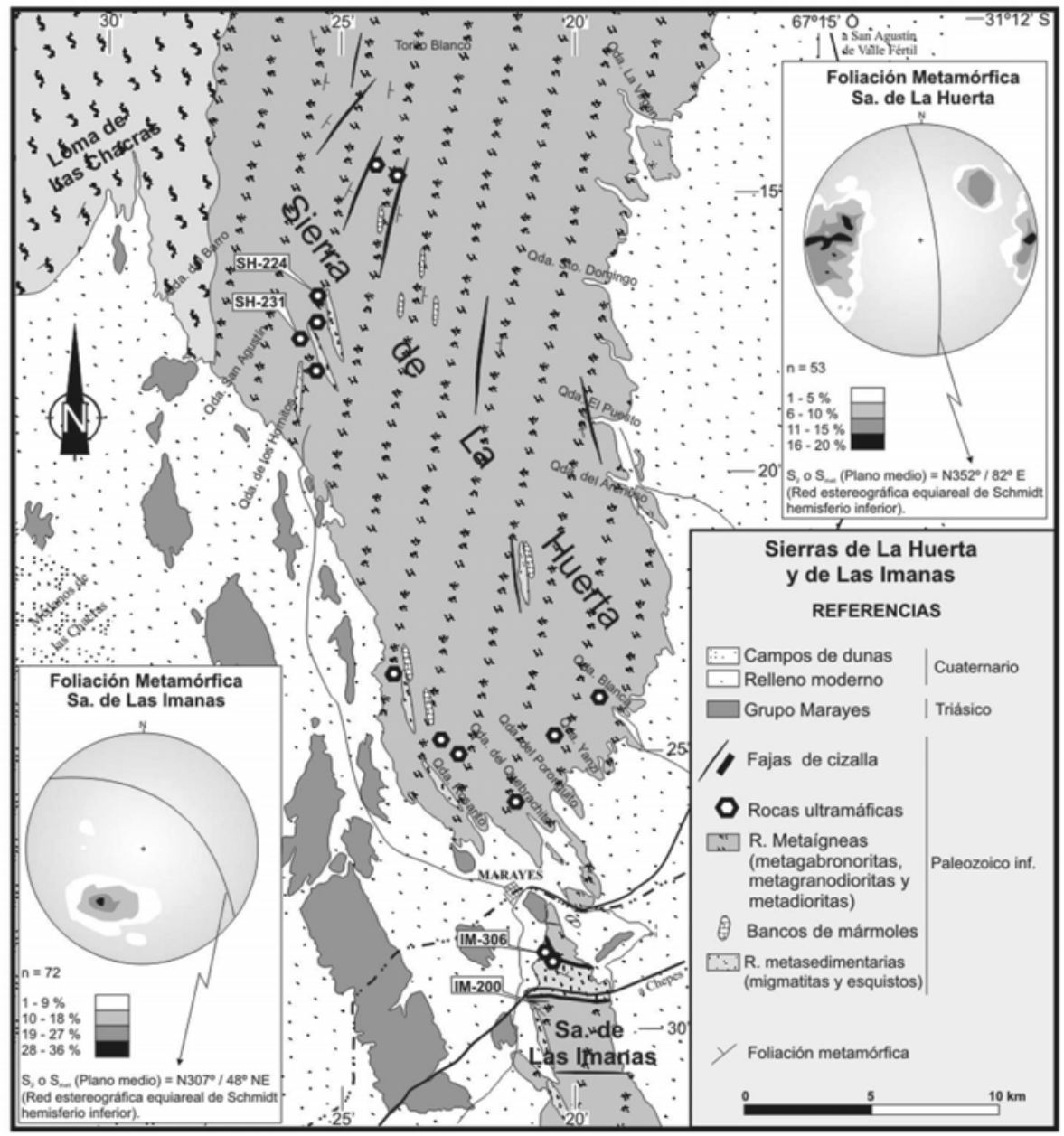

FIG. 2. Mapa geológico de la sierra de La Huerta y del sector norte de la sierra de Las Imanas (modificado de Murra, 2004).

Fértil (SVF) central, en donde se han realizado dataciones sobre gabros y granodioritas con características geoquímicas, mineralógicas y texturales equiparables a las del sector de la SI-SLH. En la sierra de Valle Fértil existen edades de cristalización de $478 \pm 4$ Ma para las unidades básicas (gabros hornbléndicos), y de $470 \pm 5$ Ma para las unidades intermedias (granodioritas calcoalcalinas, edades U-Pb SHRIMP sobre circones, Pankhurst et al., 2000). En el sector central de la SVF, se obtuvieron edades U-Pb SHRIMP en los bordes de circones provenientes de migmatitas granatíferas de $465,9 \pm 4,4 \mathrm{Ma}$, y en diatexitas con granate-cordierita de $466,5 \pm 7,7 \mathrm{Ma}$, las que se adjudican a la edad mínima del clímax metamórfico producido durante la Orogenia Famatiniana (Rapela et al., 2001). Estas edades son equivalentes a la edad Sm/Nd de 465 \pm 4 Ma obtenida sobre granate+roca total en migmatitas del sector central de la SI (Galindo et al., 2004b).

Al este del área de estudio, en el batolito de Los Llanos-Chepes-Ulapes (Fig. 1), las edades de cristalización para el magmatismo principal granodiorítico varían entre 490 y $483 \mathrm{Ma}(\mathrm{U}-\mathrm{Pb}$ SHRIMP, Pankhurst et al., 1998 y 2000; Sims et al., 1998), mientras que la edad del clímax metamórfico de $479 \pm 4 \mathrm{Ma}(\mathrm{U}-\mathrm{Pb}$ SHRIMP, Pankhurst et al., 2000), es deducido a partir de la edad de cristalización de los granitos anatécticos formados por la fusión de metasedimentos durante la intrusión del magmatismo granodiorítico (Dahlquist et al., 2005).

La formación de milonitas, localizadas en fajas de cizallamiento dúctil con cinemática inversa, marcaría el 
cierre de la Orogenia Famatiniana, y esta etapa se habría iniciado aproximadamente a los 460-452 Ma (Casquet et al., 2001; Casquet et al., 2003), continuado hasta los $432 \pm 4 \mathrm{Ma}$ (edad plateau ${ }^{40} \mathrm{Ar} /{ }^{39} \mathrm{Ar}$ en anfíbol, Castro de Machuca et al., 2005b).

La información geocronológica discutida indica que las unidades magmáticas y metamórficas de las
SLH-SI son la continuación austral de la sierra de Valle Fértil, y a su vez, que los procesos magmáticos y metamórficos de estos sectores son casi contemporáneos con los registrados en las sierras de Chepes y Ulapes, y por lo tanto integrables a una misma etapa orogénica de, al menos, 60 Ma de duración (Orogenia Famatiniana).

\section{CARACTERIZACIÓN PETROGRÁFICA Y QUÍMICA MINERAL DE LA UNIDAD METAÍGNEA}

Las rocas metamórficas de las sierras de La Huerta y Las Imanas se las agrupa en dos unidades principales (Fig. 2): Unidad metaígnea y Unidad metasedimentaria (migmatitas, gneises, esquistos y mármoles). La Unidad metaígnea está compuesta por ortogneises derivados de gabros, noritas, dioritas y granodioritas hornbléndicas, y por rocas ultramáficas metamorfizadas. Es la unidad de mayor extensión areal, abarcando todo el sector centro-oriental de la SLH, y el sector norte y central de la SI (Fig. 2). En el presente trabajo se describen especialmente las rocas de la primera unidad.

Las muestras seleccionadas para el análisis con microsonda electrónica corresponden a una metacuarzo-norita, una metaperidotita y una metapiroxenita de la Unidad metaígnea de la SLH-SI. Los análisis se realizaron con una microsonda electrónica JEOL modelo superprobe JXA 8900-M del Centro de Microscopia Electrónica Luis Bru, dependiente de la Universidad Complutense de Madrid (España). Se trabajó en condiciones estándar para este tipo de análisis, es decir, muestras pulidas a espejo y metalizadas con grafito como conductor. El equipo se programó para una rutina normal de elementos mayoritarios, con una intensidad de corriente $15 \mathrm{Kv}, 20 \mathrm{nA}$ y 5 micras de diámetro del haz de electrones. Para la calibración se utilizó la lista de patrones en su mayoría del Smithsonian Institute (Jarosewich et al., 1980). Las correcciones ZAF se realizaron automáticamente mediante el software HP-NX del equipo. Los análisis químicos de elementos mayoritarios, minoritarios, trazas y tierras raras fueron realizados en ACTLABS (Canadá) por ICP-ICP/MS.

\section{METACUARZO-NORITAS Y METADIORITAS}

Son rocas de grano medio, color gris oscuro y equigranulares, que afloran en forma de lentes subredondeados de unos 2 a $3 \mathrm{~m}$ de longitud. No se observan cumulatos, lentes o bandeados por diferenciación, sino que predomina el aspecto macizo, y normalmente con una foliación metamórfica dada por la orientación de biotita y anfíbol, cuyo plano medio es $\mathrm{N} 352^{\circ} / 82^{\circ} \mathrm{E}$ para la $\mathrm{SLH}$, mientras que en la SI se observa una rotación de la foliación hacia el oeste, siendo el plano medio de N307\%/48 NE (Fig. 2).

Las rocas máficas de composición básica e intermedia de la SVF (gabros, noritas, dioritas y tonalitas) han sido caracterizadas geoquímicamente por Pankhurst et al. (2000). Presentan rangos de $\mathrm{SiO}_{2}$ del 40 al $63 \%$, con $\mathrm{K}_{2} \mathrm{O}$ que no supera el $1 \%$, índices de saturación en alúmina (ISA) 0,55 a 1, Rb/Sr=0,020,15, $\Sigma T R=39-126$ ppm, Eu/Eu* $=1, y[\mathrm{La} / \mathrm{Yb}]_{n}$ entre 2 y 4 . Además, presentan relaciones iniciales ${ }^{87} \mathrm{Sr} /$ ${ }^{86} \mathrm{Sr}=0,706-0,710$ (similares a los granitoides del sector central de la sierra de Chepes, Pankhurst et al., 2000), y $\varepsilon N d$ de -2,4 a -6. Estas signaturas reflejan una fuente isotópicamente evolucionada como lo es la corteza media a inferior o el manto litosférico. Los modelos petrogenéticos propuestos plantean importante fusión de una sección completa de la corteza y del manto Proterozoico (Pankhurst et al., 2000). La muestra IM-200 (Tabla 1, Fig. 2) es representativa de las metacuarzo-noritas de la SI (31²9'51"S, $\left.67^{\circ} 20^{\prime} 59^{\prime \prime} \mathrm{W}\right)$. Geoquímicamente, tiene un contenido de $\mathrm{SiO}_{2}$ del 63\%, con $\mathrm{K}_{2} \mathrm{O}=0,44 \%$, (ISA) $=0,61, \mathrm{Rb} /$ $\mathrm{Sr}=0,03$, y una $\Sigma \mathrm{TR}=117 \mathrm{ppm}$. Presenta una leve anomalía de $\mathrm{Eu}\left(\mathrm{Eu} / \mathrm{Eu}^{\star}=0,95\right)$, y un bajo fraccionamiento de las tierras raras pesadas $\left([\mathrm{La} / \mathrm{Yb}]_{n}=8,4\right)$. Estas características geoquímicas sugieren una equivalencia con las rocas máficas de la SVF, perteneciendo por lo tantoal mismo ambiente del arco Famatiniano.

En general, las rocas metaígneas máficas de la SI presentan una textura blastogranular con transiciones a granolepidoblástica. A esta unidad se la puede subdividir en dos grupos principales, de acuerdo a la 
TABLA 1. ANÁLISIS QUíMICOS REPRESENTATIVOS DE ROCAS METAÍGNEAS MÁFICAS Y ULTRAMÁFICAS DE LA SIERRA DE LAS IMANAS.

\begin{tabular}{|c|c|c|c|c|c|c|c|c|c|c|c|c|c|c|c|c|c|}
\hline Ox Ma (\%peso) & $\mathrm{SiO}_{2}$ & $\mathrm{TiO}_{2}$ & $\mathrm{Al}_{2} \mathrm{O}_{3}$ & $\mathrm{Fe}_{2} \mathrm{O}_{3}$ & MnO & MgO & $\mathrm{CaO}$ & $\mathrm{Na}_{2} \mathrm{O}$ & $\mathrm{K}_{2} \mathrm{O}$ & $\mathrm{P}_{2} \mathrm{O}_{5}$ & LOI & Total & $\# \mathrm{Mg}$ & Q & A & $\mathbf{P}$ & M \\
\hline MetaQtz-norita IM-200 & 63,42 & 0,91 & 14,98 & 7,63 & 0,15 & 2,37 & 6,18 & 2,61 & 0,44 & 0,22 & 0,74 & 99,65 & 37,91 & 17,75 & 0,008 & 82,25 & 38,00 \\
\hline Metaperidotita IM-306 & 48,28 & 0,44 & 5,42 & 8,43 & 0,16 & 12,78 & 17,70 & 0,56 & 0,24 & 0,04 & 5,09 & 99,13 & 75,12 & & & & 100,00 \\
\hline Elem. traza (ppm) & $\mathbf{R b}$ & $\mathrm{Sr}$ & La & $\mathrm{Ce}$ & Pr & Nd & $\mathrm{Sm}$ & $\mathrm{Eu}$ & Gd & Tb & Dy & Ho & Er & $\mathrm{Tm}$ & Yb & Lu & $\mathrm{Cr}$ \\
\hline etaQtz-norita IM-200 & 6,00 & 227,00 & 21,60 & 51,10 & 5,75 & 22,00 & 4,37 & 1,25 & 3,69 & 0,54 & 2,87 & 0,57 & 1,76 & 0,26 & 1,73 & 0,25 & $n / d$ \\
\hline Metaperidotita IM-306 & 7,00 & 71,00 & 3,32 & 9,02 & 1,19 & 5,74 & 1,61 & 0,44 & 1,89 & 0,33 & 1,88 & 0,38 & 1,16 & 0,16 & 1,02 & 0,131 & 1680,00 \\
\hline
\end{tabular}

Los elementos mayoritarios fueron determinados con ICP y los elementos traza con ICP-MS en los laboratorios de ACTLABs, Ontario, Canadá. Hierro total calculado como $\mathrm{Fe}_{2} \mathrm{O}_{3}$. $\mathbf{n} / \mathbf{d}=$ =no determinado; $\mathbf{Q}, \mathbf{A}, \mathbf{P}$ : parámetros de clasificación modal; $\mathbf{M}$ : porcentaje modal de $\mathrm{mafitos}$. $\# \mathbf{M g}=\mathrm{Mg}^{+2} / \mathrm{Mg}^{+2}+\mathrm{Fe}^{+2}$.

presencia o no de piroxenos (gabros y noritas, o dioritas, tonalitas y granodioritas, respectivamente). Las asociaciones minerales representativas son (simbología mineral según Kretz, 1983): Metacuarzo-

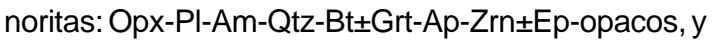
en metadioritas: PI-Am-Qtz-Bt-Ep-Ap-Zrn-opacos. La descripción petrográfica y caracterización de la química mineral que se realiza a continuación está referida específicamente a las metacuarzo-noritas.

El ortopiroxeno se encuentra en cristales anhedrales de 1 a $3 \mathrm{~mm}$, y corresponde esencialmente a $\mathrm{En}_{59-61}{ }^{-}$ $\mathrm{Fs}_{37-39}-\mathrm{Wo}_{0.7-0.8}$ (cf. Tabla 2). El cuarzo se presenta en cristales anhedrales de variados tamaños $(0,2-2 \mathrm{~mm})$. La plagioclasa se diferencia en, al menos, dos tipos petrográficos y composicionales (cf. Tabla 3) que pueden correlacionarse con etapas respectivas en la evolución genética de la roca. $\mathrm{PI}_{1}\left(\mathrm{An}_{82-87}\right)$, son cristales tabulares anhedrales a subhedrales, de 0,1 a 0,5 mm, saussuritizados, y en ocasiones incluidos en el ortopiroxeno. Esta plagioclasa es interpretada como perteneciente a la etapa de cristalización magmática de la roca. $\mathrm{La} \mathrm{PI}_{2}\left(\mathrm{An}_{52}\right)$ tiene características similares a la anterior pero de mucho mayor tamaño (hasta 2 $\mathrm{mm}$ ), por sectores formando agregados granoblásticos y asociados a la biotita. $\mathrm{La} \mathrm{PI}_{2}$ sería el producto de una recristalización durante la etapa metamórfica.

Con respecto a los anfíboles, se pueden diferenciar dos grupos principales (cf. Tabla 4): I) Anfíboles cálcicos, con $(\mathrm{Ca}+\mathrm{Na})_{\mathrm{B}=}$ 1,86 a.p.f.u., $\mathrm{X}_{\mathrm{Mg}}=0,62$ a.p.f.u., $(\mathrm{Na}+\mathrm{K})_{A=} 0,29$ a.p.f.u., $\mathrm{Ca}_{A=} 0$ a.p.f.u., clasificándose como tschermakitasaHbl-tschermakitas; y II)Anfíboles ferromagnesianos, con $(\mathrm{Ca}+\mathrm{Na})_{\mathrm{B}=} 0,16$ a.p.f.u., y $\mathrm{X}_{\mathrm{Fe}}=0,79$ a.p.f.u., clasificándose como Mgcummingtonitas (Leake et al., 1997). La observación petrográfica permite, además, diferenciar dentro del primer grupo tres tipos con características ópticas y texturales distintas. $\mathrm{Hbl}_{1}$, en cristales euhedrales y subhedrales, en ocasiones incluidos en plagioclasa
(Fig. 3) y de tamaños de 0,1 a 0,3 mm, sugiriendo que se trataría de un anfíbol primario (magmático). $\mathrm{Hbl}_{2}$, más abundante y de mayor tamaño que el primero ( 0,5 a $1 \mathrm{~mm}$ ), de origen blástico y asociado a $\mathrm{Pl}_{2}$ y al granate (Fig. 4), presentándose en ocasiones como blastos de tipo poiquilítico y con inclusiones de plagioclasa y cuarzo. También es claramente blástica y post-magmática. $\mathrm{Hbl}_{3}$ se presenta en agregados de cristales mucho más pequeños que los anteriores $(<0,1 \mathrm{~mm})$, anhedrales y asociados a biotita y minerales opacos. Se trataría del anfíbol cálcico más tardío de la asociación metamórfica. El segundo grupo de anfíboles corresponde a cummingtonitas, y se lo observa principalmente en las muestras de la quebrada de Los Hornitos (Fig. 2). Es de hábito fibroso, incoloroy asociado de forma coronítica al ortopiroxeno junto con magnetita(Fig. 5). En la asociación coronítica se observa un núcleo de ortopiroxeno, rodeado de cummingtonita y ésta, a su vez, rodeada parcialmente por el anfíbol cálcico $\left(\mathrm{Hbl}_{3}\right)$ y la magnetita. En la parte más externa se observa plagioclasa que también interviene en la reacción. La cummingtonita es menos frecuente que los anfíboles anteriores, pero su presencia es muy importante para acotar las condiciones físicas de una de las etapas de retrogradación metamórfica.

La biotita (en porcentajes variables) presenta relaciones $\mathrm{X}_{\mathrm{Fe}}=0,32-0,34 ; \mathrm{TiO}_{2}=2,76-3,17 \%$ (cf. Tabla 5), por sectores es abundante y en otros se encuentra como mineral accesorio. La mayoría de las veces se la observa asociada a los anfíboles cálcicos (hornblendas) y minerales opacos, y suele marcar la foliación de la roca.

El granate es un mineral poco frecuente y escaso en las rocas metaígneas máficas, encontrándose en sectores muy localizados y sin una distribución uniforme en la roca. Es rico en almandino, piropoy grosularia $\left(\mathrm{Alm}_{49-54}-\mathrm{Prp}_{22}-\mathrm{Grs}_{10-12}-\mathrm{Sps}_{8-12}-\mathrm{Adr}_{3-4}\right.$, Tabla 6). 


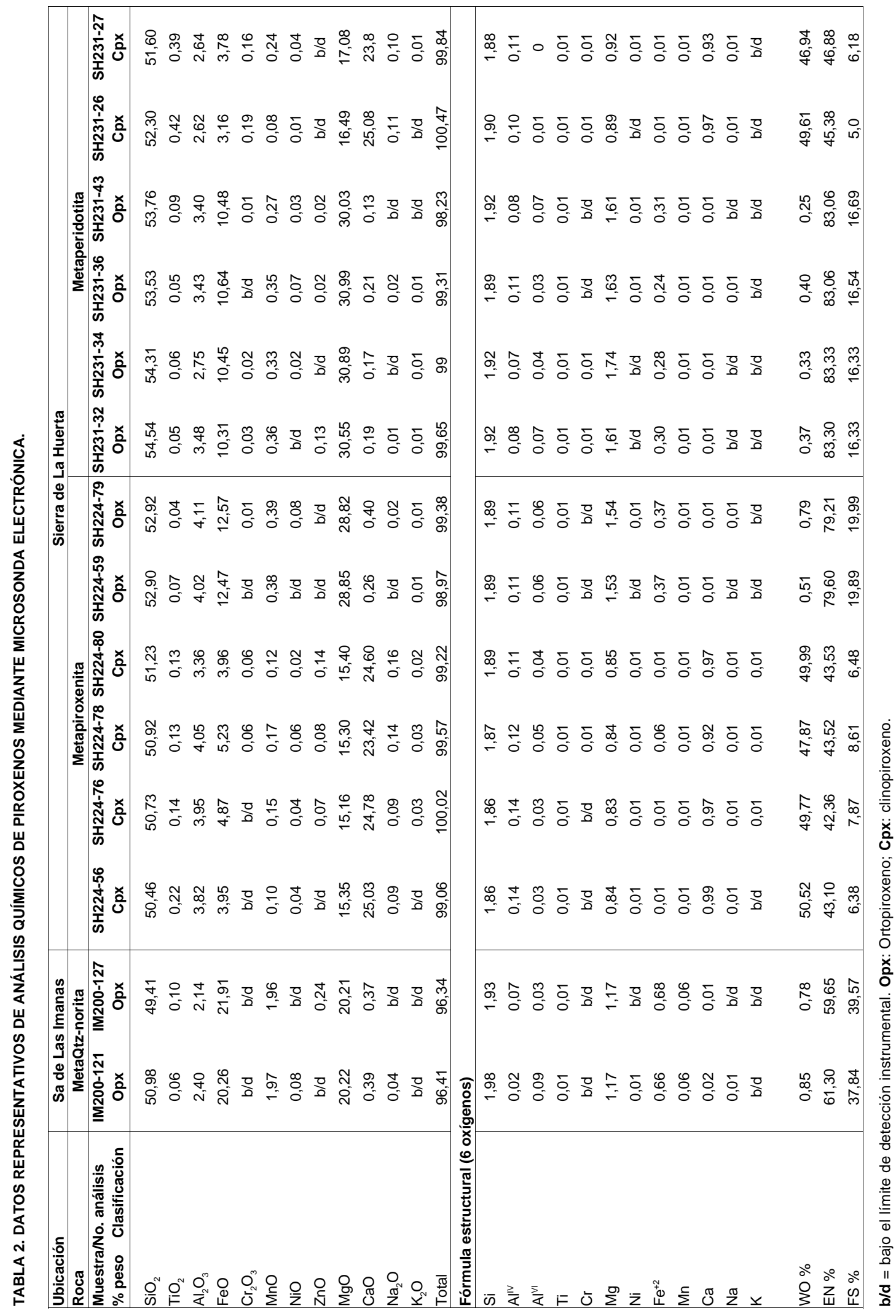


TABLA 3. DATOS REPRESENTATIVOS DE ANÁLISIS QUÍMICOS DE PLAGIOCLASAS MEDIANTE MICROSONDA ELECTRÓNICA.

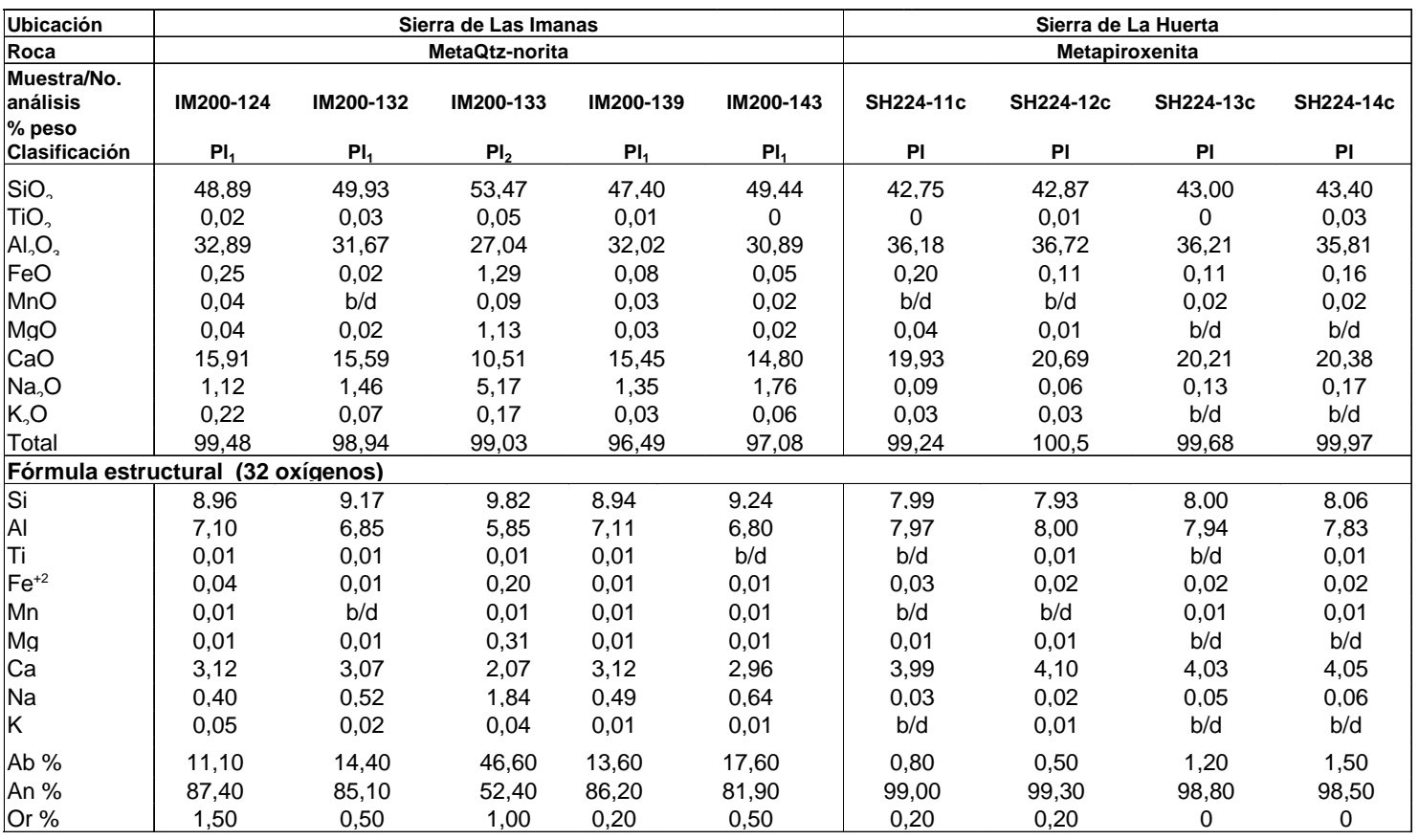

b/d = bajo el límite de detección instrumental. PI: plagioclasa.

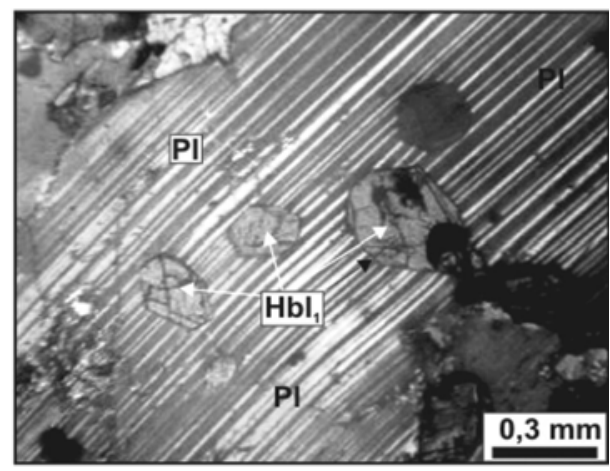

FIG. 3. Metacuarzo-norita con cristales euhedrales de $\mathrm{Hbl}_{1}$ incluidos en plagioclasa.

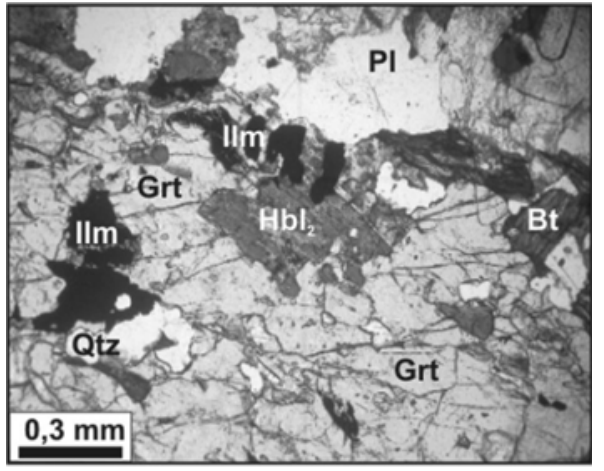

FIG. 4. Contexto textural de la paragénesis (2), PI+Qtz+Am \pm Ilm \pm Grt, formada durante el evento metamórfico M1 y según la reacción [1].

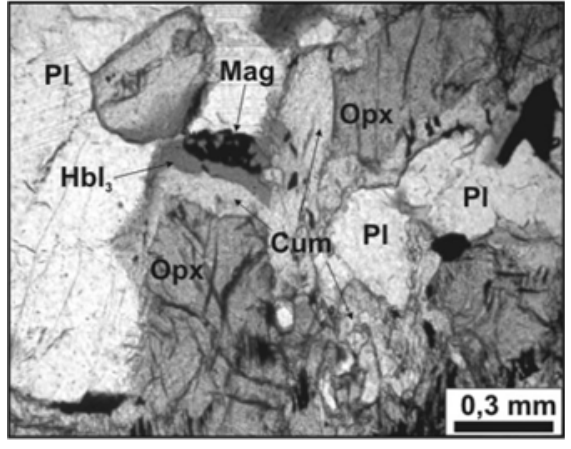

FIG. 5. Texturas coroníticas del M2 con formación de cummingtonita en la metacuarzo-norita según la reacción [3]. 


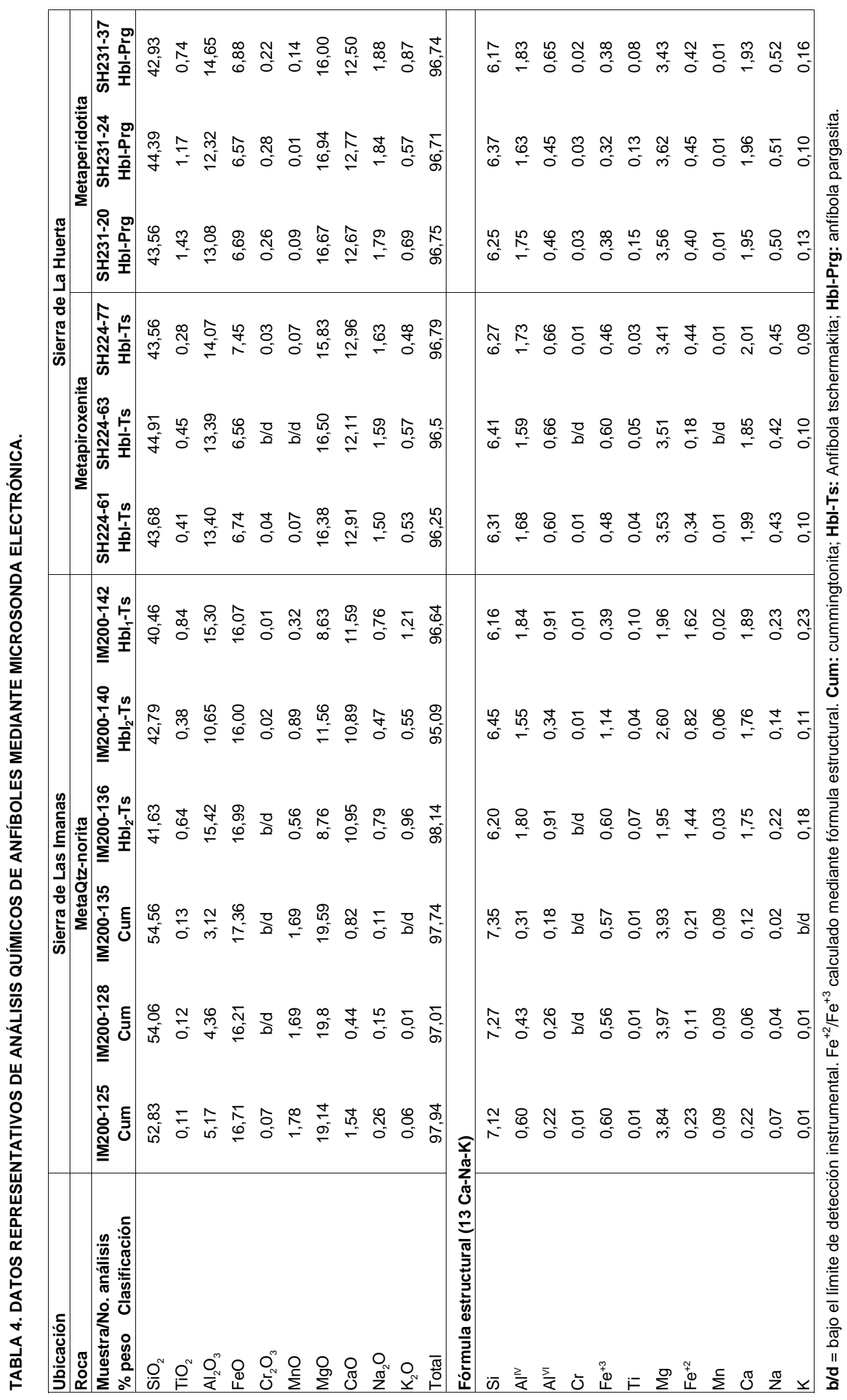


TABLA 5.DATOS REPRESENTATIVOS DE ANÁLISIS QUÍMICOS DE BIOTITAS MEDIANTE MICROSONDA ELECTRÓNICA.

\begin{tabular}{|c|c|c|c|c|c|}
\hline \multirow{3}{*}{\begin{tabular}{|l|} 
Ublcaclón \\
Roca \\
Muestra/No. \\
análisis \\
$\%$ peso \\
Clasificación
\end{tabular}} & \multirow{2}{*}{\multicolumn{3}{|c|}{$\begin{array}{l}\text { Sa. de Las Imanas } \\
\text { MetaQtz-norita }\end{array}$}} & \multirow{2}{*}{\multicolumn{2}{|c|}{$\begin{array}{l}\text { Sa. de La Huerta } \\
\text { Metaperidotita }\end{array}$}} \\
\hline & & & & & \\
\hline & IM200-130 & IM200-131 & IM200-134 & $\begin{array}{c}\text { SH231-51 } \\
\text { Phl } \\
\end{array}$ & $\begin{array}{c}\text { SH231-52 } \\
\text { Phl } \\
\end{array}$ \\
\hline $\mathrm{SiO}_{2}$ & 33,51 & 34,38 & 34,97 & 38,54 & 38,64 \\
\hline $\mathrm{TiO}_{2}$ & 3,17 & 2,97 & 2,76 & 0,97 & 0,88 \\
\hline $\mathrm{Al}_{2} \mathrm{O}_{3}$ & 20,60 & 16,06 & 16,47 & 17,04 & 17,12 \\
\hline $\mathrm{Cr}_{2} \mathrm{O}_{3}$ & 0,03 & 0,06 & $b / d$ & $b / d$ & 0,01 \\
\hline $\mathrm{FeO}$ & 12,46 & 12,46 & 13,97 & 4,30 & 4,26 \\
\hline $\mathrm{MnO}$ & 0,08 & 0.17 & 0,21 & $b / d$ & 0,03 \\
\hline MgO & 13,81 & 14,71 & 14,97 & 22,43 & 22,44 \\
\hline Zno & 0,14 & 0,19 & 0,03 & $b / d$ & $b / d$ \\
\hline $\mathrm{NiO}$ & 0,04 & 0,07 & 0,03 & 0,18 & 0,08 \\
\hline $\mathrm{CaO}$ & 0,11 & 0,02 & 0.04 & $b / d$ & 0,04 \\
\hline $\mathrm{Na}_{2} \mathrm{O}$ & 0,09 & 0,12 & 0,12 & 0,81 & 0,81 \\
\hline $\mathrm{K}_{2} \mathrm{O}$ & 8,91 & 9,46 & 9,25 & 8,64 & 8,62 \\
\hline Total & 94,95 & 92,58 & 94,79 & 92,93 & 92,96 \\
\hline \multicolumn{6}{|c|}{ Fórmula estructural (22 oxígenos) } \\
\hline$\overline{\mathrm{Si}}$ & 5,30 & 5,63 & 5,61 & 5,57 & 5,58 \\
\hline $\mathrm{Al}^{\mathrm{IV}}$ & 2,70 & 2,37 & 2,39 & 2,43 & 2,42 \\
\hline$\left.A\right|^{v \prime}$ & 1,14 & 0,72 & 0,73 & 0,47 & 0,49 \\
\hline $\mathrm{Ti}$ & 0,38 & 0,37 & 0,33 & 0,11 & 0,09 \\
\hline $\mathrm{Fe}^{+2}$ & 1,65 & 1.71 & 1,88 & 0,52 & 0,51 \\
\hline $\mathrm{Cr}$ & 0,01 & 0,01 & $b / d$ & $b / d$ & 0,01 \\
\hline Mn & 0,01 & 0,02 & 0,03 & $b / d$ & 0,01 \\
\hline $\mathrm{Mg}$ & 3,26 & 3,59 & 3,58 & 4,83 & 4,83 \\
\hline $\mathrm{Zn}$ & 0,02 & 0,02 & $b / d$ & $\mathrm{~b} / \mathrm{d}$ & $b / d$ \\
\hline $\mathrm{Ni}$ & $\mathrm{b} / \mathrm{d}$ & 0.01 & $\mathrm{~b} / \mathrm{d}$ & 0,02 & 0,01 \\
\hline $\mathrm{Ca}$ & 0,02 & 0,01 & 0,01 & $b / d$ & 0,01 \\
\hline $\mathrm{Na}$ & 0,03 & 0,04 & 0,04 & 0,23 & 0,23 \\
\hline K & 1,80 & 1.97 & 1,89 & 1,59 & 1,59 \\
\hline $\mathrm{Fe} / \mathrm{FeMg}$ & 0,34 & 0,32 & 0,34 & 0,10 & 0,10 \\
\hline
\end{tabular}

\section{ROCAS ULTRAMÁFICAS (METAPIROXENITAS Y METAPERIDOTITAS)}

En distintos sectores de las sierras de La Huerta y Las Imanas se localizan pequeños cuerpos de rocas ultramáficas-ultrabásicas (piroxenitas y peridotitas) (Castro de Machuca et al., 1996 y 2002; Vujovich et al., 1996; Murra y Baldo, 2004a) que están asociadas a las metacuarzo-noritas, y afloran en forma de lentes de 2 a $10 \mathrm{~m}$ de longitud dispuestos de manera concordante con la foliación regional. Los principales afloramientos reconocidos y muestreados se pueden observar en la figura 2, al igual que otros citados por distintos autores.

El estudio geoquímico de las rocas ultramáficas de la SLH realizado por Castro de Machuca et al. (1996), sugiere un origen residual vinculado al magmatismo básico de la SVF-SLH-SI. Presentan valores de $\mathrm{SiO}_{2}$ $\sim 38-48 \%$, con $\mathrm{CaO} \sim 8-16 \%$ y álcalis muy bajos $(0,19-$ 1,19\%). El promedio de $\mathrm{Cr}$ es de 987 ppm.

La muestra IM-306 (Tabla 1) representaría una

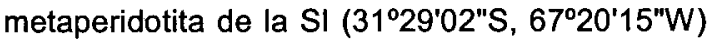

TABLA 6.DATOS REPRESENTATIVOS DE ANALISIS QUIMICOS DE GRANATES MEDIANTE MICROSONDA ELECTRONICA

\begin{tabular}{|c|c|c|}
\hline \multirow{2}{*}{\begin{tabular}{|l|} 
Roca \\
Muestra/No. análisis \\
$\%$ peso Clasiflcación \\
\end{tabular}} & \multicolumn{2}{|c|}{ MetaQtz-norita } \\
\hline & $\begin{array}{c}\text { IM200-141 } \\
\text { Grt }\end{array}$ & $\begin{array}{c}\text { IM200-145 } \\
\text { Grt }\end{array}$ \\
\hline $\mathrm{SiO}_{2}$ & 38,19 & 38,32 \\
\hline $\mathrm{TiO}_{2}$ & 0,12 & 0,18 \\
\hline $\mathrm{Al}_{2} \mathrm{O}_{3}$ & 21,72 & 21,42 \\
\hline $\mathrm{Cr}_{2} \mathrm{O}_{3}$ & $\mathrm{~b} / \mathrm{d}$ & 0,05 \\
\hline $\mathrm{FeO}$ & 24,96 & 23,02 \\
\hline $\mathrm{MnO}$ & 3,54 & 5,26 \\
\hline $\mathrm{MgO}$ & 5,41 & 5,51 \\
\hline $\mathrm{CaO}$ & 5,00 & 5,75 \\
\hline Total & 99,05 & 99,52 \\
\hline \multicolumn{3}{|c|}{ Fórmula estructural (24 oxígenos) } \\
\hline $\mathrm{Si}$ & 6,04 & 6,02 \\
\hline$A \|^{v \prime}$ & 4.05 & 3,96 \\
\hline $\mathrm{Fe}^{+3}$ & 0,16 & 0,15 \\
\hline $\mathrm{Ti}$ & 0,01 & 0,02 \\
\hline $\mathrm{Cr}$ & $\mathrm{b} / \mathrm{d}$ & 0,01 \\
\hline $\mathrm{Fe}^{+2}$ & 3,14 & 2,87 \\
\hline $\mathrm{Mg}$ & 1,28 & 1,29 \\
\hline Mn & 0,47 & 0,70 \\
\hline $\mathrm{Ca}$ & 0,85 & 0,97 \\
\hline Almandino & 54,69 & 49,26 \\
\hline Andradita & 4,32 & 3,89 \\
\hline Grosularia & 10,46 & 12,54 \\
\hline Piropo & 22,26 & 22,13 \\
\hline Espesartina & 8,28 & 12,01 \\
\hline $\begin{array}{l}\text { Uvarovita } \\
\text { b/d=bajo ellimite de dete } \\
\text { estructural. Grt: granate. }\end{array}$ & $\frac{0}{\text { trumental. } F}$ & $\frac{0,16}{\text { mediante }}$ \\
\hline
\end{tabular}

con la siguiente composición: $\mathrm{SiO}_{2}=48 \%, \mathrm{CaO}=$ $17,7 \%$, suma de álcalis $=0,8$ y concentraciones importantes de $\mathrm{Cr}(1680 \mathrm{ppm})$. El contenido de $\mathrm{SiO}_{2}$ no se corresponde con el de una peridotita, indicando que el evento metamórfico habria modificado la composición original del protolito.

El análisis petrográfico permite diferenciar dos grupos con características texturales y mineralógicas diferentes: rocas ultramáficas olivínicas, y ultramáficas piroxénicas. El primer grupo presenta una asociación mineralógica y texturas relícticas que permiten identificarla como una metaperidotita (Iherzolita), en tanto que el segundo correspondería a una metapiroxenita (websterita), tal como fuera reconocido por Castro de Machuca et al. (1996). Las metaperidotitas presentan la siguiente asociación mineral:

OI-Opx-Cpx-Am-Spl-Srp $\pm I I m \pm M a g \pm P y \pm P n \pm P h I$

Lamuestra representativa de las metaperidotitas, y que ha sido utilizada para caracterizar la química 
mineral, es la SH-231, ubicada en el sector de la quebrada de Los Hornitos (Fig. 2; 31¹9'21"S, $\left.67^{\circ} 25^{\prime} 34^{\prime \prime} \mathrm{W}\right)$.

El ortopiroxeno, el clinopiroxeno y el anfíbol son los minerales máficos que componen el $80 \%$ de la roca, mientras que los relictos de olivino serpentinizados más los minerales opacos conforman el resto. Los cristales relícticos de piroxenos ( $1 \mathrm{~mm})$, están en parte recristalizados y normalmente rodean al olivino, que se encuentra parcial o totalmente serpentinizado. Tanto el clinopiroxeno como el ortopiroxeno y el olivinoconstituyen laasociación mineral primaria relíctica. Los ortopiroxenos relícticos ígneos son cristales anhedrales de tamaños de 1 a $3 \mathrm{~mm}$, muy fracturados, y se clasifican como enstatita $\left(E n_{83-84}\right.$, Tabla 2), con un contenido en Al variable entre 0,07-0,11 a.p.f.u. En muchos sectores se encuentra rodeado (o incluido) por los porfidoblastos de anfíbol.

Los clinopiroxenos analizados se clasifican como diópsidos $\left(\mathrm{Wo}_{46-50}, \mathrm{En}_{45-47}, \mathrm{Fs}_{5-6}\right.$, Tabla 2). Se presentan como inclusiones en anfíboles, de formas subhedrales $\mathrm{y}$ anhedrales de alrededor de 0,3 mm. En ocasiones se observan exoluciones de minerales opacos dispuestos según las dos direcciones del clivaje basal de los piroxenos. El olivino presenta el hábito característico de cristales subredondeados y fracturas serpentinizadas. Es una $\mathrm{Fo}_{84}$, pobre en $\mathrm{NiO}(0,16-$ 0,28\%) y de composición uniforme (cf. Tabla 7).

El anfíbol se presenta como porfiroblastos poiquilíticos (>5 mm) incluyendo al resto de la asociación mineral. Son anfíboles cálcicos (cf. Tabla4) con $\mathrm{X}_{\mathrm{Mg}}$ $>$ 0,5, TSi=6,1 y 6,3 a.p.f.u., clasificándose como pargasitas a Hbl-pargasitas (Leake et al., 1997).

Se observa, además, óxidos, sulfuros de hierro y hierro-níquel (pentlanditas con 40,9\% de NiO). Los más abundantes son los minerales del grupo de los espinelos, presentándose frecuentemente como exsoluciones junto con IIm+Mag en diópsido y en anfíboles. Los espinelos de las metaperidotitas (cf. Tabla 8), muestran un $\mathrm{X}_{\mathrm{Mg}}$ variable entre 1,14y 2,03 y sobre la base del porcentaje de hercinita, se pueden diferenciar 2 grupos: el primero $\left(\mathrm{Spl}_{1}\right)$, es relativamente pobre en $\mathrm{Fe}^{+2}$, con $29-33 \%$ de $\mathrm{Hc}$, y de hábitos euhedrales y subhedrales, asociado a magnetita, ilmenita y pirita. Esta asociación de óxidos podría asignarse a la etapa magmática de la roca. En el segundo grupo $\left(\mathrm{Spl}_{2}\right)$, el porcentaje de $\mathrm{Hc}$ sube al 38-44\% y presentan un $1,5 \%$ de $\mathrm{Cr}_{2} \mathrm{O}_{3} \mathrm{La}$ composición química del resto de los minerales opacos en las metaperidotitas ha sido incluida en la tabla 9.

Las metapiroxenitas coroníticas han sido muestreadas en la SLH en el sector central, a la altura
TABLA 7.DATOS REPRESENTATIVOS DE ANÁLISIS QUÍMICOS DE OLIVINOS MEDIANTE MICROSONDA ELECTRÓNICA.

\begin{tabular}{|lcccc|}
\hline Ubicación & \multicolumn{4}{c|}{ Sa. de La Huerta } \\
\hline Roca & \multicolumn{4}{c|}{ Metaperidotita } \\
\hline $\begin{array}{l}\text { Muestra/ } \\
\text { No. análisis }\end{array}$ & SH231-25 & SH231-33 & SH231-15c & SH231-16c \\
\% peso Clasificación & Ol & Ol & Ol & Ol \\
\hline $\mathrm{SiO}_{2}$ & 39,00 & 39,44 & 40,06 & 39,42 \\
$\mathrm{TiO}_{2}$ & $\mathrm{~b} / \mathrm{d}$ & $\mathrm{b} / \mathrm{d}$ & 0,02 & $\mathrm{~b} / \mathrm{d}$ \\
$\mathrm{Al}_{2} \mathrm{O}_{3}$ & 0,01 & 0,01 & 0,02 & $\mathrm{~b} / \mathrm{d}$ \\
$\mathrm{FeO}$ & 15,18 & 14,83 & 14,99 & 15,23 \\
$\mathrm{MnO}$ & 0,28 & 0,29 & 0,24 & 0,24 \\
$\mathrm{MgO}$ & 44,59 & 44,13 & 45,51 & 44,44 \\
$\mathrm{NiO}$ & 0,17 & 0,18 & 0,28 & 0,16 \\
$\mathrm{Total}$ & 99,23 & 98,91 & 101,14 & 99,5 \\
\hline $\mathrm{Fórmula} \mathrm{estructural}$ & $(4$ oxígenos) & & & \\
\hline $\mathrm{Si}$ & 1,00 & 1,00 & 0,99 & 1,00 \\
$\mathrm{Al}$ & $\mathrm{b} / \mathrm{d}$ & $\mathrm{b} / \mathrm{d}$ & 0,01 & $\mathrm{~b} / \mathrm{d}$ \\
$\mathrm{Ti}$ & $\mathrm{b} / \mathrm{d}$ & $\mathrm{b} / \mathrm{d}$ & $\mathrm{b} / \mathrm{d}$ & $\mathrm{b} / \mathrm{d}$ \\
$\mathrm{Fe}{ }^{+2}$ & 0,32 & 0,31 & 0,31 & 0,32 \\
$\mathrm{Mn}$ & 0,01 & 0,01 & 0,01 & 0,01 \\
$\mathrm{Mg}$ & 1,70 & 1,67 & 1,69 & 1,68 \\
$\mathrm{Ni}$ & 0,01 & 0,01 & 0,01 & 0,01 \\
& & & & \\
$\mathrm{Fe} / \mathrm{Fe}+\mathrm{Mg}$ & 0,16 & 0,16 & 0,16 & 0,16 \\
\hline
\end{tabular}

b/d: bajo el límite de detección instrumental.

de la quebrada El Puesto, y en el sector occidental en la quebrada de Los Hornitos (Fig. 2). La muestra analizada con microsonda electrónica es la SH-224 ubicada en este último sector (31018'07"S, 67025'37"W). Laasociación mineral de esta roca ultramáfica es:

Opx-Cpx-Spl-Am $\pm \mathrm{Pl} \pm \mathrm{Mag} \pm \mathrm{IIm}$

El ortopiroxeno $\left(\mathrm{En}_{79}\right.$, con 0,17 Al a.p.f.u., Tabla 2) es de color rosado pleocroico y se presenta en agregados granoblásticos anhedrales a subhedrales de 0,2 a $0,5 \mathrm{~mm}$.

El anfíbol es una variedad cálcica y se clasifica como Hbl-tschermakita a Hbl-pargasita $\left(X_{M g}>0,5 y\right.$ rangos de TSi entre 6,27 y 6,41, Tabla 4). Se presenta como un agregado granoblástico de blastos anhedrales y como porfidoblastos poiquilíticos que incluyen al resto de la asociación mineral.

El clinopiroxeno se presenta en cristales relícticos de 0,5 a $2 \mathrm{~mm}$, poiquilítico y anhedral o como un intercrecimiento simplectítico con espinelo. En ambos casos se clasifica como diópsido $\left(\mathrm{Hd}_{12-16}\right.$, Tabla 2), con un\% $\mathrm{CaO}=23-25, \mathrm{y} \mathrm{X}_{\mathrm{Fe}}=0,04$.

La plagioclasa $\left(A n_{98,5-99,4}\right)$ es sumamente escasa, en cristales anhedrales y por lo general sericitizada. La poca cantidad actual de plagioclasa se debería al consumo de ésta durante la formación de clinopiroxeno, y especialmente anfíbol generado durante la etapa de retromorfismo. 
El espinelo $\left(\mathrm{Hc}_{36-40}\right)$ se lo observa vinculado al desarrollo de la textura simplectítica (de formas lamelares y globulares), y como cristales de mayor tamaño y anhedrales incluidos en el anfíbol. El contenido en $\mathrm{Cr}_{2} \mathrm{O}_{3}$ es muy bajo $(0,01 \%)$, el de $\mathrm{NiO}$ no supera el $0,5 \%(0,1-0,3 \%)$, mientras que el $\mathrm{ZnO}$ presenta porcentajes aún menores $(0,2 \%$, Tabla 8$)$. Los bajos tenores de estos óxidos concuerdan con su origen metamórfico más que magmático.

TABLA 8. DATOS REPRESENTATIVOS DE ANÁLISIS QUÍMICOS DE ESPINELOS MEDIANTE MICROSONDA ELECTRÓNICA.

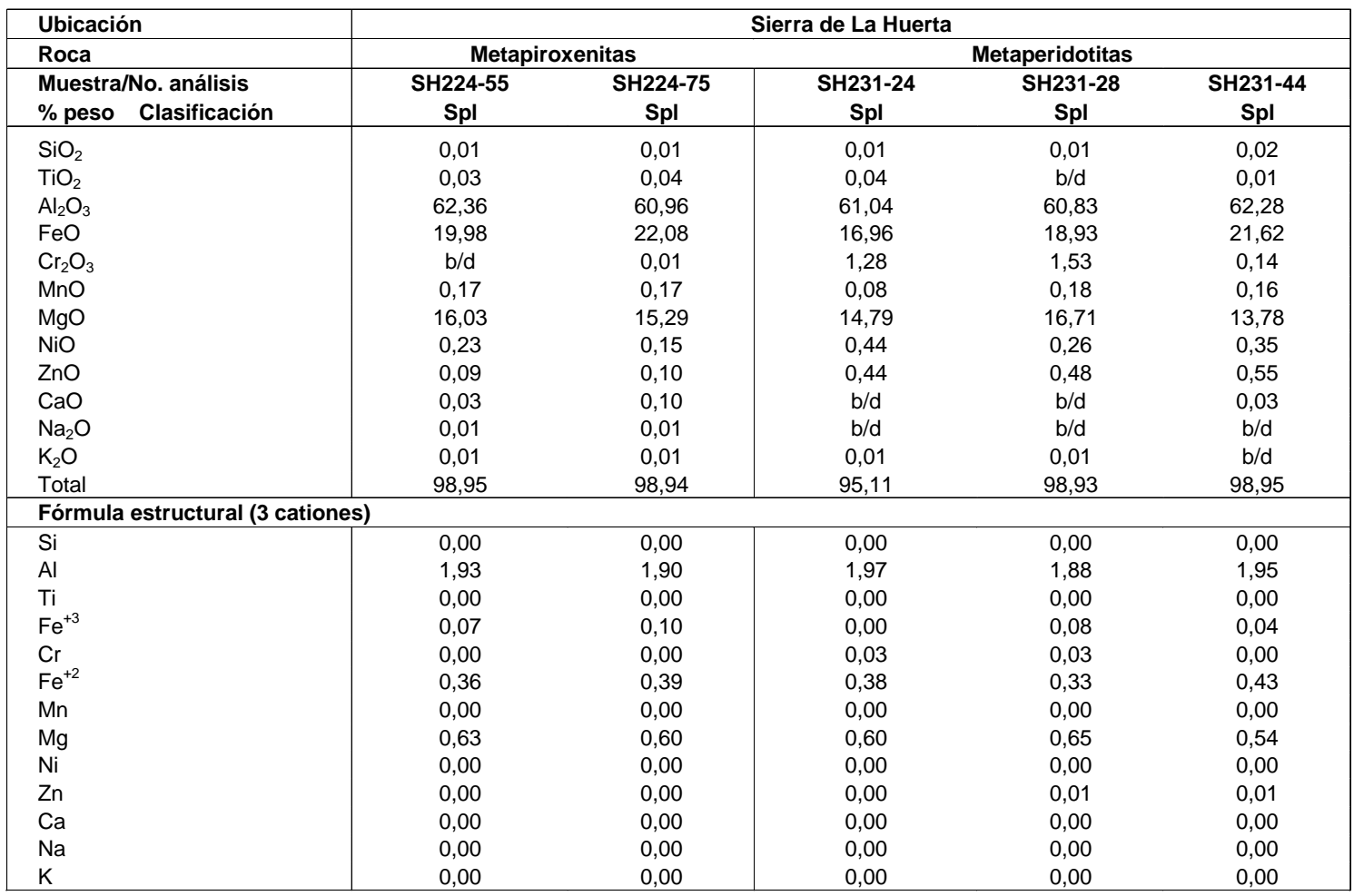

b/d = bajo el límite de detección instrumental. $\mathrm{Fe}^{+3}$ calculado a partir de la fórmula estructural. Spl: espinelo.

\section{TERMOBAROMETRÍA DE LAS UNIDADES METAÍGNEAS MÁFICAS}

En las metacuarzo-noritas, es posible diferenciar 3 asociaciones minerales principales:

(1) $-\mathrm{Pl}_{1}+\mathrm{Opx}+\mathrm{Hbl}_{1}+$ opacos-Asociación ígnea (relíctica)

(2) $-\mathrm{Pl}_{2}+\mathrm{Grt}+\mathrm{Hbl}_{2}+\mathrm{Qtz}+\mathrm{llm}-\mathrm{Paragénesis} \mathrm{metamórfica}$ M1 (climax metamórfico)

(3) - Cum+Hbl + Qtz+Mag-Paragénesis metamórfica M2 (retrógrado)

La asociación (1) (Fig. 6) indicaría que la composición original podría corresponder a rocas noríticas o gabro-noríticas. La paragénesis (2) (Fig. 4), se genera a partir de la reacción:
$\mathrm{PI}+\mathrm{Hbl}_{2}=\mathrm{Grt}+\mathrm{Qtz}+\mathrm{IIm}+\mathrm{H}_{2} \mathrm{O}$

[1]

indicando condiciones de grado alto (facies de granulita) para el evento M1.

La etapa de retrogradación M2 está caracterizada por el desarrollo de una textura coronítica que genera la asociación (3) (Fig. 5). Para esta etapa, las reacciones propuestas son:

$\mathrm{Opx}+\mathrm{PI}+\mathrm{IIm}+\mathrm{H}_{2} \mathrm{O}=\mathrm{Hbl}_{3}+\mathrm{Qtz}+\mathrm{Mag}$

$\mathrm{Opx}+\mathrm{Qtz}+\mathrm{H}_{2} \mathrm{O}=\mathrm{Cum}+\mathrm{Mag}$ 
La etapa M2 está dentro del grado medio del metamorfismo (facies de anfibolita). Reacciones similares a [2] y [3] han sido propuestas por Graessner y
Schenk (2001), para explicar la retrogradación del macizo de Sila (norte de Calabria, Italia), pero estos autores las plantean sin la presencia de magnetita.

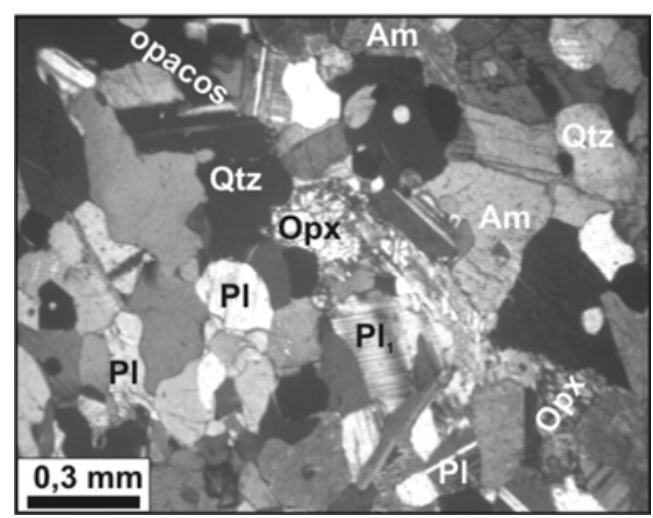

FIG. 6. Dominio textural de las metacuarzo-noritas con preservación de la textura ígnea. Se observa a la plagioclasa subhedral junto al ortopiroxeno.

TABLA 9. DATOS REPRESENTATIVOS DE ANÁLISIS QUÍMICOS DE MAGNETITAS, SULFUROS E ILMENITA MEDIANTE MICROSONDA ELECTRÓNICA.

\begin{tabular}{|c|c|c|c|c|c|c|}
\hline \multirow{2}{*}{\begin{tabular}{|l|} 
Ubicación \\
Roca \\
Muestra/No. análisis \\
\end{tabular}} & \multirow{3}{*}{$\begin{array}{c}\text { SI } \\
\text { MetaQtz-norita } \\
\text { IM200-126 } \\
\text { Mag }\end{array}$} & \multicolumn{5}{|c|}{ Sa. de La Huerta } \\
\hline & & \multirow{2}{*}{$\begin{array}{c}\text { Metapirox. } \\
\text { SH224-71 } \\
\text { Mag }\end{array}$} & \multicolumn{4}{|c|}{ Metaperidotita } \\
\hline \begin{tabular}{|ll} 
Muestra/No. análisis \\
$\%$ peso $\quad$ Clasificación
\end{tabular} & & & $\begin{array}{c}\text { SH231-26 } \\
\text { Mag }\end{array}$ & $\begin{array}{c}\text { SH231-31 } \\
\text { Py }\end{array}$ & $\begin{array}{c}\text { SH231-30 } \\
\text { Pn }\end{array}$ & $\begin{array}{c}\mathrm{SH} 231-27 \\
\mathrm{IIm}\end{array}$ \\
\hline $\mathrm{SiO}_{2}$ & 0,83 & 0,63 & 0,01 & 0,04 & 0,30 & 0,73 \\
\hline $\mathrm{TiO}_{2}$ & 0,19 & 0,31 & 0,27 & $\mathrm{~b} / \mathrm{d}$ & $b / d$ & 52,58 \\
\hline $\mathrm{Al}_{2} \mathrm{O}_{3}$ & 0,82 & 1,38 & 0,41 & 0,01 & 0,06 & 0,92 \\
\hline $\mathrm{FeO}$ & 87,51 & 84,71 & 88,03 & 75,28 & 41,44 & 36,81 \\
\hline $\mathrm{Fe}_{2} \mathrm{O}_{3}$ & 0 & 0 & 0 & 0 & 0 & 0 \\
\hline $\mathrm{Cr}_{2} \mathrm{O}_{3}$ & 0,03 & 0,04 & 0,83 & 0,01 & 0,01 & 0,05 \\
\hline $\mathrm{MnO}$ & 0,09 & 0,02 & 0,05 & 0,03 & 0,04 & 2,74 \\
\hline $\mathrm{MgO}$ & 0,37 & 0,86 & 0,36 & 0,01 & 0,31 & 5,33 \\
\hline $\mathrm{NiO}$ & $b / d$ & 0,11 & 0,27 & 0,32 & 40,93 & 0,07 \\
\hline $\mathrm{ZnO}$ & $b / d$ & 0,05 & $b / d$ & 0,11 & 0,03 & $b / d$ \\
\hline $\mathrm{CaO}$ & 0,29 & 0,14 & 0,02 & $\mathrm{~b} / \mathrm{d}$ & $\mathrm{b} / \mathrm{d}$ & $b / d$ \\
\hline $\mathrm{Na}_{2} \mathrm{O}$ & 0,01 & 0,04 & $b / d$ & 0,01 & 0,01 & 0,01 \\
\hline Total & 90,16 & 88,32 & 90,25 & 75,80 & 83,17 & 99,24 \\
\hline \multicolumn{7}{|c|}{ Fórmula estructural (Mag = 3 cationes; IIm = 2 cationes) } \\
\hline Si & 0,03 & 0,03 & 0,00 & & & 0,02 \\
\hline Al & 0,04 & 0,07 & 0,02 & & & 0,03 \\
\hline $\mathrm{Ti}$ & 0,01 & 0,01 & 0,01 & & & 0,76 \\
\hline $\mathrm{Fe}^{+3}$ & 1,88 & 1,86 & 1,94 & & & 0 \\
\hline $\mathrm{Cr}$ & 0,00 & 0,00 & 0,03 & & & $\mathrm{~b} / \mathrm{d}$ \\
\hline $\mathrm{Fe}^{+2}$ & 1,00 & 0,97 & 0,98 & & & 0,75 \\
\hline Mn & 0,00 & 0,00 & 0,00 & & & 0,06 \\
\hline $\mathrm{Mg}$ & 0,02 & 0,05 & 0,02 & & & 0,19 \\
\hline $\mathrm{Ni}$ & 0,00 & 0,00 & 0,01 & & & $\mathrm{~b} / \mathrm{d}$ \\
\hline$Z n$ & 0,00 & 0,00 & 0,00 & & & $\mathrm{~b} / \mathrm{d}$ \\
\hline $\mathrm{Ca}$ & 0,01 & 0,01 & 0,00 & & & $\mathrm{~b} / \mathrm{d}$ \\
\hline $\mathrm{Na}$ & 0,00 & 0,00 & 0,00 & & & 0 \\
\hline
\end{tabular}

b/d = bajo el límite de detección instrumental. $\mathrm{Fe}^{+3}$ calculado a partir de la fórmula estructural. Mag: magnetita; Py: pirita; Pn: pentandlita; IIm: ilmenita. 


\section{ESTIMACIÓN DE LAS CONDICIONES DEL PICO METAMÓRFICO (M1)}

La paragénesis (2), $\mathrm{Pl}_{2}+\mathrm{Grt}+\mathrm{Hbl}_{2}+\mathrm{Qtz} \pm$ Ilm, permite el uso combinado del par hornblenda-plagioclasa (Holland y Blundy, 1994), y granate-hornblenda (Graham y Powell, 1984). Se ha empleado el programa "Hbl-Pl" desarrollado por Holland y Blundy (1994), que permite conocer la temperatura de equilibrio de cada reacción a diferentes presiones $(0,5,10$ y 15 kbar). Los rangos de $\mathrm{T}^{\mathrm{o}}$ obtenidos son similares para cada uno de los métodos aplicados. Para el termómetro $\mathrm{Hbl}-\mathrm{PI}$, los valores de temperatura obtenidos fueron entre 701 y $730^{\circ} \mathrm{C}$ (análisis representativos Pl: IM200-143-Am: IM200-142, Tablas 3 y 4, respectivamente), mientras que el par granate-hornblenda arrojó valores de 690 a $730^{\circ} \mathrm{C}$ (análisis Am: IM200-
142; Grt: IM200-141; Am: IM200-142; Grt: IM200145, Tablas 4 y 6 , respectivamente, y Tabla 10).

Para el cálculo de la presión, se utilizó también la combinación de dos geobarómetros. El primero se basa en el contenido de Al en anfíbol, utilizando las calibraciones de Hollister et al. (1987). Los valores máximos y mínimos obtenidos son de 7,9 y 8,1 kbar (análisis Am: IM200-136, IM200-142; Tabla 4). El segundo método utilizado es el geobarómetro granate-hornblenda-plagioclasa-cuarzo (Kohn y Spear, 1989), obteniendo valores levemente superiores, $\mathrm{P} \approx 8,4-8,9$ kbar (análisis PI: IM200-143; Am: IM200142; Grt: IM200-145; Tablas 3, 4 y 6, respectivamente y Tabla 10).

TABLA 10. DATOS P-T PARA LAS DIFERENTES ASOCIACIONES EN LAS ROCAS METAÍGNEAS MÁFICAS Y ULTRAMÁFICAS DE LA SLH Y SI.

\begin{tabular}{|c|c|c|c|c|c|c|}
\hline Litología/muestra & Asociación & Geotermómetro & $\begin{array}{c}\text { Temperatura } \\
\left({ }^{\circ} \mathrm{C}\right)\end{array}$ & Geobarómetro & $\begin{array}{c}\text { Presión } \\
\text { (Kbar) }\end{array}$ & Interpretación \\
\hline MetaQtz-norita/IM200 & $\mathrm{Pl}_{2}-\mathrm{Grt}-\mathrm{Hbl}_{2}-\mathrm{Qtz}$ & $\mathrm{Hbl}-\mathrm{Pl}(1)$ & $701-730^{\circ} \mathrm{C}$ & Al en Am (2) & $\begin{array}{c}7,9-8,1 \\
k b \\
8,4-8,9\end{array}$ & M1 \\
\hline MetaQtz-norita/IM200 & $\mathrm{Pl}_{2}-\mathrm{Grt}-\mathrm{Hbl}_{2}-\mathrm{Qtz}$ & Grt - Hbl (3) & $690-730^{\circ} \mathrm{C}$ & Grt - Hbl - PI - Qtz (4) & $\begin{array}{c}\mathrm{kb} \\
4,5-5,9\end{array}$ & M1 \\
\hline MetaQtz-norita/IM200 & Cum - $\mathrm{Hbl}_{3}-\mathrm{Qtz}$ & $\mathrm{Hbl}-\mathrm{Pl}(1)$ & $605-620^{\circ} \mathrm{C}$ & Al en Am (2) & $\begin{array}{c}k b \\
3,9 \pm 2,3\end{array}$ & M2 \\
\hline MetaQtz-norita/IM200 & Cum - $\mathrm{Hbl}_{3}-\mathrm{Qtz}$ & THERMOCALC (5) & $566 \pm 53^{\circ} \mathrm{C}$ & THERMOCALC (5) & $\mathrm{kb}$ & M2 \\
\hline Metaperidotita/SH231 & Ol - Opx - Cpx - Spl - Am & Ol - Spl (6) & $726^{\circ} \mathrm{C}$ & & & M2 \\
\hline Metaperidotita/SH231 & Ol - Opx - Cpx - Spl - Am & OI - Spl - Opx (7) & $700^{\circ} \mathrm{C}$ & & & M2 \\
\hline Metaperidotita/SH231 & Ol - Opx - Cpx - Spl - Am & Cpx - Opx (8) & $750^{\circ} \mathrm{C}$ & & & M2 \\
\hline Metapiroxenita/SH224 & Opx - PI - Cpx - Am - Spl & Cpx - Opx (8) & $730^{\circ} \mathrm{C}$ & & & M2 \\
\hline Metapiroxenita/SH224 & Opx - PI - Cpx - Am - Spl & $\mathrm{Hbl}-\mathrm{Pl}(1)$ & $729 \pm 52{ }^{\circ} \mathrm{C}$ & & & M2 \\
\hline
\end{tabular}

(1) Holland y Blundy, 1994; (2) Hollister et al., 1987; (3) Graham y Powell, 1984; (4) Kohn y Spear, 1989; (5) Powell y Holland, 1988; (6) Roeder et al., 1977; (7) Gasparik y Newton, 1984; (8) Wood y Banno, 1973.

\section{ESTIMACIONES DE LAS CONDICIONES DE RETROGRADACIÓN METAMÓRFICA (M2)}

Para la paragénesis coronítica (3) $\mathrm{Cum}+\mathrm{Hbl}_{3}+$ Qtz \pm Mag, donde además permanece la plagioclasa como fase en equilibrio, las condiciones de presión y temperatura se determinaron utilizando nuevamente el geotermómetro hornblenda-plagioclasa de Holland y Blundy (1994), obteniéndose temperaturas de 605 a $620 \pm 40^{\circ} \mathrm{C}$ (análisis PI: IM200-133; Am: IM200136; Tablas 3 y 4, respectivamente y Tabla 10), y la presión para esta temperatura fue calculada en base al contenido de $\mathrm{Al}$ en la $\mathrm{Hbl}_{3}$ dando valores entre 4,5 y 5,9 kbar (análisis Am: IM-140; Tablas 4 y 10).
También se calcularon las condiciones P-T mediante el programa THERMOCALC (Powell y Holland, 1988), con el cual se obtuvieron valores sensiblemente menores, pero dentro de los límites de error. Estos valores son de $566 \pm 53^{\circ} \mathrm{C}$ para la temperatura

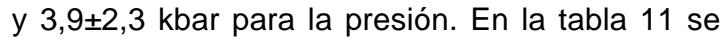
puede observar con que minerales/miembros finales se realizaron los cálculos de este último método (análisis Opx: IM200-127; PI: IM200-132; Cum: IM200-135; Hbl: IM200-140; Tablas 2, 3 y 4, respectivamente). 
TABLA 11. DATOS UTILIZADOS PARA LOS CÁLCULOS CON EL PROGRAMA THERMOCALC.

\begin{tabular}{|c|c|c|c|c|c|c|c|}
\hline \multicolumn{2}{|c|}{ Ortopiroxeno } & \multicolumn{2}{|c|}{ Anfíboles } & \multicolumn{2}{|c|}{ Plagioclasa } & \multicolumn{2}{|c|}{ Resultados } \\
\hline $\begin{array}{c}\text { Muestra } \\
\text { Etapa P-T }\end{array}$ & $\begin{array}{c}\text { IM-200 } \\
\text { M2 }\end{array}$ & $\begin{array}{c}\text { Muestra } \\
\text { Etapa P-T }\end{array}$ & IM-200 M2 & $\begin{array}{c}\text { Muestra } \\
\text { Etapa P-T }\end{array}$ & $\begin{array}{c}\mathrm{IM}-200 \\
\text { M2 }\end{array}$ & $\begin{array}{c}\text { Muestra } \\
\text { Etapa P-T }\end{array}$ & $\begin{array}{c}\text { IM-200 } \\
\text { M2 }\end{array}$ \\
\hline $\begin{array}{l}\text { En } \\
\text { Fs }\end{array}$ & $\begin{array}{l}0,35 \\
0,12\end{array}$ & $\begin{array}{l}\text { Mg-Ts } \\
\text { Cum } \\
\text { Gru } \\
\text { Fe-Act } \\
\text { Gln }\end{array}$ & $\begin{array}{l}0,016 \\
0,13 \\
0,0023 \\
0,00163 \\
0,00853\end{array}$ & $\begin{array}{l}A b \\
A n\end{array}$ & $\begin{array}{l}0,32 \\
0,88\end{array}$ & $\begin{array}{l}\text { aH2O } \\
\mathrm{T}^{\circ} \mathrm{C} \\
\text { s.d (T) } \\
\mathrm{P} \mathrm{kb} \\
\text { s.d (P) } \\
\text { Correl. } \\
\text { Fit. } \\
\mathrm{Nr}\end{array}$ & $\begin{array}{l}1 \\
566 \\
53 \\
3,9 \\
2,3 \\
0,786 \\
1,2 \\
0\end{array}$ \\
\hline
\end{tabular}

\section{TERMOBAROMETRÍA DE LAS UNIDADES METAÍGNEAS ULTRAMÁFICAS}

En las rocas ultramáficas clasificadas como metaperidotitas, la formación de los porfiroblastos de anfíboles que incluyen al resto de la asociación anhidra, sugiere una reacción de tipo (Fig. 7):

$$
\mathrm{OI}+\mathrm{Cpx}+\mathrm{Opx}+\mathrm{H}_{2} \mathrm{O}=\mathrm{Spl}_{2}+\mathrm{Am}
$$

Para etapas retrógradas en facies de esquistos verdes, se genera la serpentina y la magnetita a partir de las siguientes reacciones (Bucher y Frey, 1994):

$$
\begin{aligned}
& \mathrm{Ol}(\mathrm{Fa})+\mathrm{O}_{2}=\mathrm{Mag}+\mathrm{SiO}_{2} \\
& \mathrm{Ol}(\mathrm{Fo})+\mathrm{Qtz}+\mathrm{H}_{2} \mathrm{O}=\mathrm{Srp}
\end{aligned}
$$

La reacción [5] oxida el componente fayalítico del olivino formándose magnetita, y además genera la sílice $\left(\mathrm{SiO}_{2}\right)$ necesaria para que reaccione con el componente forsterítico $\left(+\mathrm{H}_{2} \mathrm{O}\right)$ en la reacción [6], y de como resultado la serpentinización del olivino.

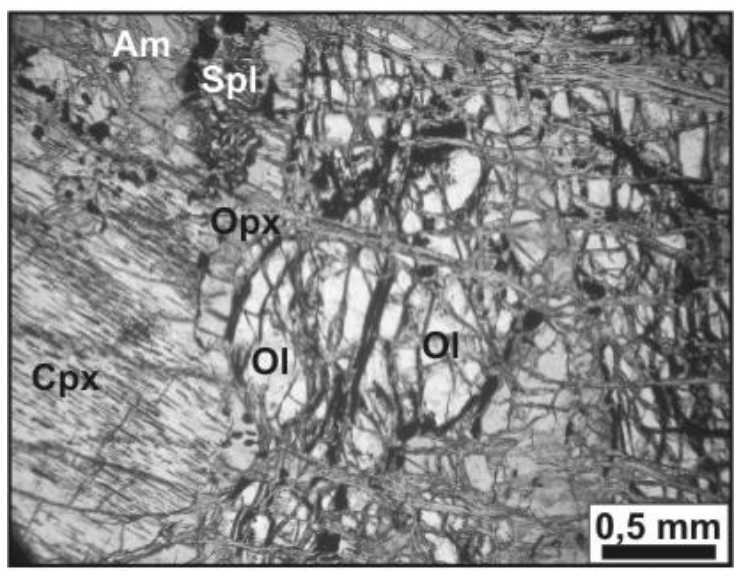

En las metapiroxenitas, las texturas relícticas preservadas dentro de los porfiroblastos de anfíboles, permiten deducir al menos un evento metamórfico previo a la formación del anfíbol. La figura 8 sintetiza las principales observaciones texturales de esta roca. Se reconocen los siguientes dominios texturales: 1. Ortopiroxenos anhedrales localizados en el núcleo de los porfiroblastos de anfíbol. 2. Simplectitas de espinelos+clionopiroxenos, también incluidos totalmente en los porfiroblastos de anfíboles. Los espinelos son de tipo lamelar en la simplectita con el clinopiroxeno, y están dispuestos perpendicularmente al contacto con la plagioclasa, en tanto que en contacto con el anfíbol, éstos son de tipo globular. 3El anfíbol suele ser un gran porfiroblasto con continuidad óptica que engloba a toda la asociación y termina bruscamente en el contacto con la plagioclasa. La interpretación evolutiva de esta textura es la siguiente:

I. Metamorfismo M1, formación de una paragénesis anhidra en condiciones granulíticas a partir de la reacción:

$\mathrm{Opx}+\mathrm{Pl}=\mathrm{Cpx}+\mathrm{Spl}_{1}$

II. Metamorfismo M2, formación de una nueva paragénesis en facies de anfibolitas según la reacción:

$\mathrm{Cpx}+\mathrm{PI}+\mathrm{Opx}+\mathrm{H}_{2} \mathrm{O}=\mathrm{Am}+\mathrm{Spl}_{2}$

La "lectura" textural de estas rocas suele brindar

FIG. 7. Asociación Opx-Cpx-Am-Spl-OI de las metaperidotitas de la SLH-SI. Contexto textural de la reacción [4]. 


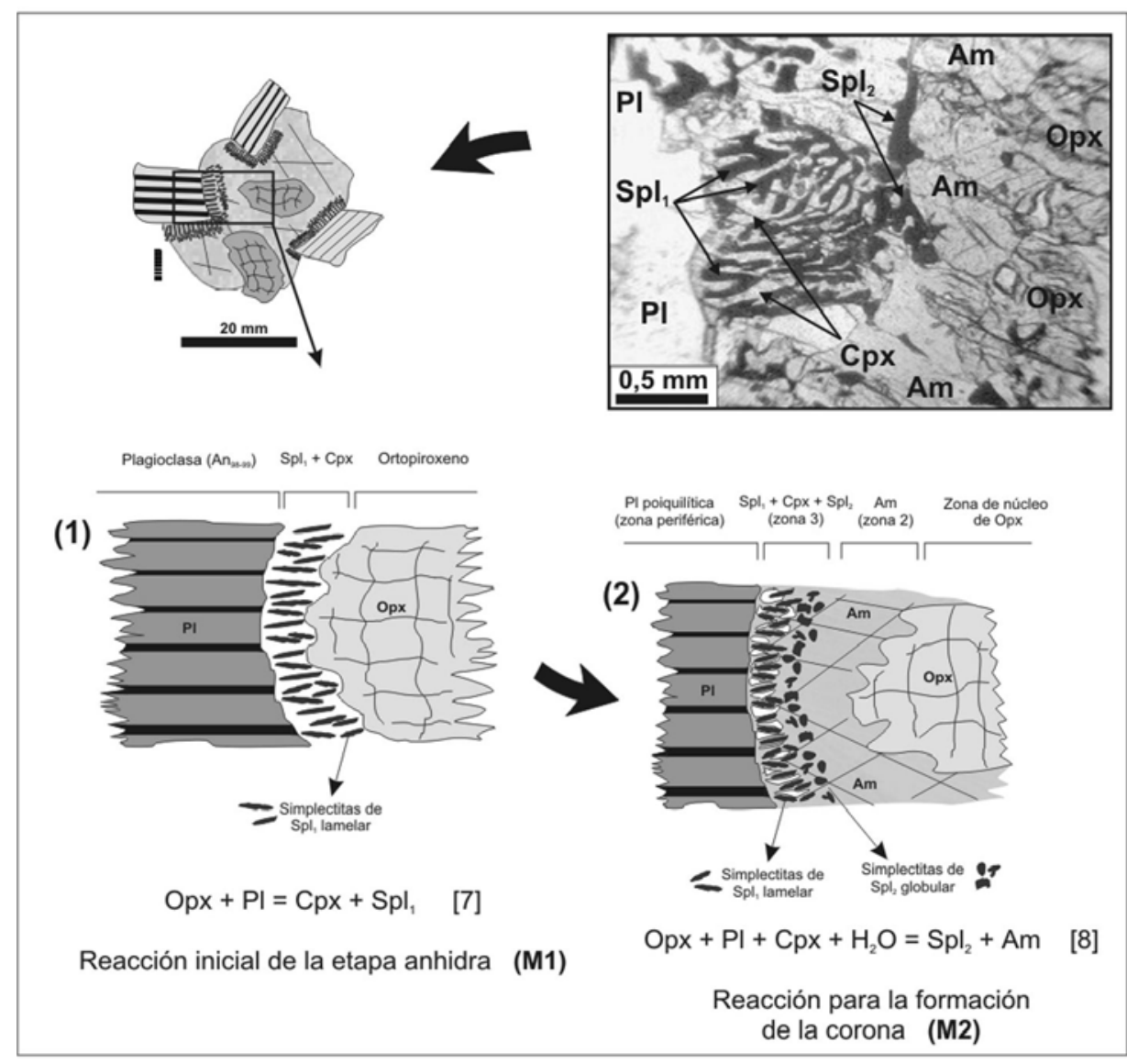

FIG. 8. Fotomicrografía del contexto textural de la corona kelifítica en las metapiroxenitas formadas a partir de las reacciones [7] y [8], y esquemas de la misma.

información cualitativa sobre su evolución termobárica. Sin embargo, la cuantificación de las condiciones de presión y temperatura de cada una de las diferentes etapas de su evolución metamórfica es, normalmente, difícil de obtener debido a que las paragénesis de minerales máficos reajustan fácilmente su composición, reequilibrándose al último evento de la evolución termobárica (Schmädicke, 2000). A pesar de esto, si se establecen los contextos texturales de las diferentes paragénesis minerales y se reconocen las principales texturas reaccionales es factible, en algunos casos, la obtención de una trayectoria P-T que sea significativa de su evolución. La grilla petrogenética para el sistema CMASH de Schmädicke (2000), basada en una piroxenita sin olivino de Granulitgebirge, Bohemian Massif (Fig. 9), permite estimar que, durante el M1, las metapiroxenitas de las SLH-SI habrían estado equilibradas dentro del campo divariante An$\mathrm{Spl}_{1}-\mathrm{Opx}-\mathrm{Cpx}$, y que durante el M2 retrogradó al campo divariante $\mathrm{An}-\mathrm{Spl}_{2}-\mathrm{Am}-\mathrm{Cpx}$, cortando la cur- va univariante de la reacción $\mathrm{Opx}+\mathrm{Cpx}+\mathrm{An}+\mathrm{H}_{2} \mathrm{O}$ $=$ Am+Spl.

A partir de la aplicación de los geotermómetros y geobarómetros específicos para este tipo de roca (Tabla 10), no fue posible obtener condiciones confiables del evento M1, debido a que la química mineral de las asociaciones analizadas están equilibradas al evento M2.

La temperatura del evento M2 en la metaperidotita con OI-Opx-Cpx-Am-Spl, se obtuvo mediante la aplicación de los siguientes termómetros: 1. Ol-Spl (Roeder et al., 1979; intercambio Fe-Mg entre olivino-espinelo), dando una temperatura de $726^{\circ} \mathrm{C}$ (análisis OI: SH231-25; Spl: SH231-24, Tablas 7 y 8, respectivamente). 2. Ol-Spl-Opx (Gaspariky Newton, 1984; intercambio de Al entre ortopiroxeno-espineloforsterita) obteniéndose una temperatura de $700^{\circ} \mathrm{C}$ (análisis Opx: SH231-32; OI: SH231-33; Spl: SH231-28, Tablas 2, 7 y 8, respectivamente) y $\mathbf{3}$. Cpx-Opx (Wood y Banno, 1973; intercambio de Ca 


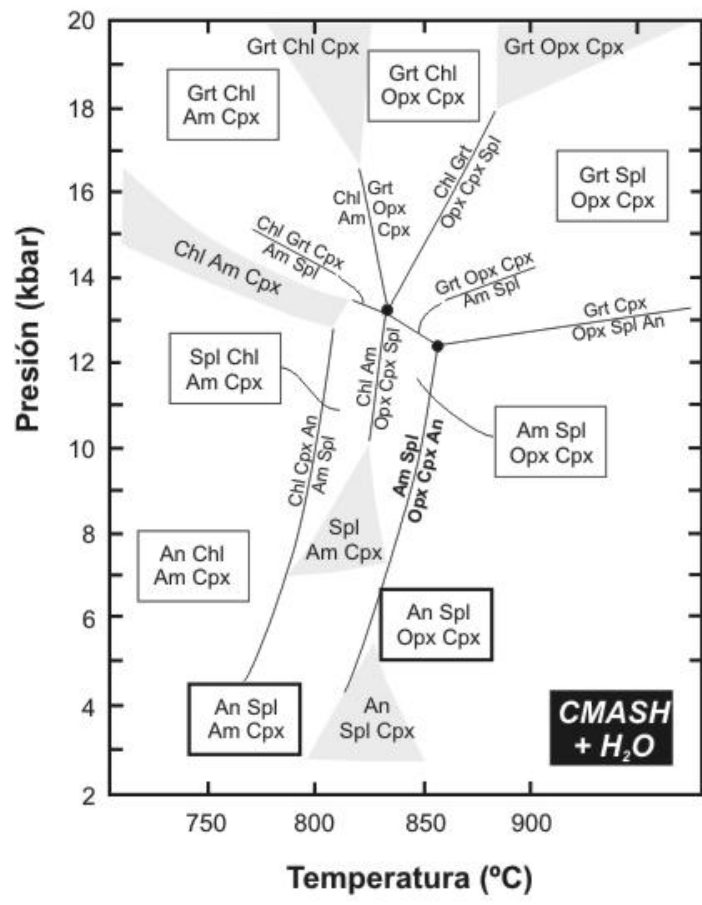

Divariante

Trivariante

FIG. 9. Diagrama de fases para rocas metapiroxénicas con una composición (\% en peso): $\mathrm{CaO}=14,1 ;(\mathrm{FeO}+\mathrm{MgO})=23,5$; $\mathrm{Al}_{2} \mathrm{O}_{3}=13,2 ; \mathrm{SiO}_{2}=43,3$ (según Schmädike, 2000). entre clinopiroxeno y ortopiroxeno), que arrojó una temperatura de $750^{\circ} \mathrm{C}$ (análisis Opx: SH231-32; Cpx: SH231-27; Tabla 2).

Para la asociación metapiroxenítica (Opx-CpxAm-PI-Spl), se utilizaron los termómetros basados en los piroxenos (Cpx-Opx) y se empleó, además, como control el termómetro Hbl-PI de Holland y Blundy (1994). Para el primer geotermómetro se obtuvo un valor de $730^{\circ} \mathrm{C}$ (análisis Opx: $\mathrm{SH} 224-79$; Cpx: SH224-80; Tabla 2), en tanto que con el segundo, un valor de $729 \pm 52^{\circ} \mathrm{C}$.

Para el cálculo de la presión se utilizó el barómetro Cpx-PI, calibrado en base al intercambio de Ca entre el clinopiroxeno y la plagioclasa (Ellis, 1980). El mejor resultado obtenido es el de 4,2 \pm 1 kbar (análisis Cpx: SH224-56; PI: SH224-12c; Tablas 2 y 3, respectivamente). Se utilizó, además, el método de multiequilibrios para calcular la estabilidad de dos reacciones específicas de la asociación metaperidotítica [4] y metapiroxenítica [8], utilizando la base de datos termodinámicos del programa TEEWQU(Berman, 1991; versión 2.02b y la base termodinámica BA96a.DAT). La figura 10 muestra que estas dos reacciones se cortan en un punto a los $720^{\circ} \mathrm{C}$ y $4,3 \mathrm{kbar}$, coincidente con el valor del barómetro de intercambio Cpx-PI, por lo tanto, consideramos a este valor de presión como representativo del evento $\mathrm{M} 2$.

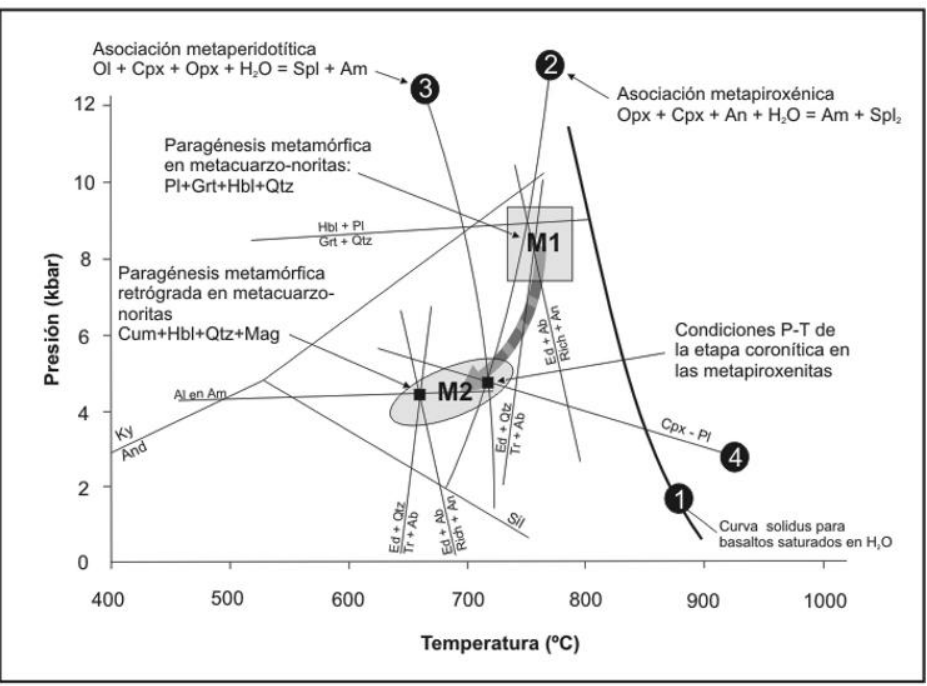

FIG. 10. Evolución P-T de la Unidad Metaígnea deducida a partir de las metacuarzo-noritas, y de las asociaciones metaperidotítica y metapiroxenítica. Curvas 2 y 3 calculadas según el TEEWQU de Berman (1991). Curva 4, barómetro de intercambio de Ca entre Cpx-PI. Modelos de actividad para cálculos TEEWQU tomados de: Fuhrman y Lindsley, 1988 (plagioclasa); Berman y Aranovich, 1996 (olivino, espinelo, piroxenos); Mader y Berman, 1992 (anfíboles). 


\section{DISCUSIÓN Y CONCLUSIONES}

La información textural y termobarométrica aportada por las unidades metaígneas de las sierras de Las Imanas y La Huerta indican que la rocas del arco magmático Famatiniano han sido metamorizadas en un primer evento metamórfico que denominamos M1, bajo condiciones de grado medio a alto $\left(710 \pm 20^{\circ} \mathrm{C}\right.$ y $\left.8,45 \pm 0,55 \mathrm{kbar}\right)$. Los valores de presión y temperatura del evento $M 1$ deducidos a partir de las unidades metaígneas, son equivalentes a los calculados a partir de las paragénesis de las rocas metasedimentarias que se encuentran intercaladas con las rocas metaígneas de las sierras de Las Imanas $\left(720-760^{\circ} \mathrm{C}\right.$ y 6,8 a 8 kbar; Murra, 2004; Murra y Baldo, en preparación). Un segundo evento metamórfico (M2) en facies de anfibolitas, formó nuevas paragénesis que están registradas en dominios texturales específicos (coronas de reacción con formación de cummingtonita, entre otras), y que permiten estimar condiciones de $620 \pm 40^{\circ} \mathrm{C}$ y $4,3 \pm 1 \mathrm{kbar}$. La trayectoria P-T deducida a partir de esto datos, indica que con posterioridad al clímax metamórfico $\mathrm{M} 1$, se produce un rápido alzamiento de $12-14 \mathrm{~km}$ que induce a una descompresión cuasi-isotérmica en las rocas del área (Fig. 10).

Castro de Machuca et al. (2004), describen coronas de reacción en metagabros de las sierra de $L a$ Huerta que generan Opx+Spl/ Cpx+Spl / Am+Spl entre olivino y plagioclasa. A partir de estas coronas, calculan temperaturas en facies de granulita de 863$930^{\circ} \mathrm{C}$ y presiones de $7-8 \mathrm{kbar}$, que son asignadas al clímax metamórfico (M1). Consideramos que los cálculos de temperatura de Castro de Machuca et al. (2004), podrian estar sensiblemente sobrevaluados debido a la dificultad de compatibilizarlos con las paragénesis de las rocas metasedimentarias (migmatitas y gneises con $\mathrm{Grt}+\mathrm{Sil}+\mathrm{Kfs}+\mathrm{Pl}+\mathrm{Bt}+\mathrm{Qtz}$ ) que afloran en bandas discontinuas, intercaladas con las unidades metaígneas, y que representan la caja de las unidades plutónicas del arco. Para temperaturas superiores a $850^{\circ} \mathrm{C}$ y presiones de $8 \mathrm{kbar}$, se debería registrar la formación de Crd+Opx+Sil, o Crd+Opx+Grt en los protolitos metapelíticos (Spear et al., 1999). Sin embargo, en las sierras de Las Imanas y La Huerta no está registrada la presencia de $\mathrm{Crd}+\mathrm{Grt} u$ Opx en las metasedimentitas. En cambio, la paragénesis $\mathrm{Bt}+\mathrm{Grt}+\mathrm{Sil}+\mathrm{K} \mathfrak{s}$, frecuente en las metapelitas de este sector, es coherente con los valores de presión y temperatura obtenidos en este trabajo.

En determinados sectores de las SLH-SI, las rocas metasedimentarias desarrollan grandes blastos de granates $(>10 \mathrm{~cm})$, que son rodeados por una corona de plagioclasa y cuarzo. Estos granates han sido utilizados para datar el metamorfismo M1, dando una edad de $465 \pm 4 \mathrm{Ma}$ (isocrona Sm-Nd, Galindo et al., 2004b; Murra, 2004). Considerando las edades de cristalización de las unidades magmáticas en la sierra Valle Fértil (470-478 Ma, Rapela et al., 2001) como representativas para las unidades del arco magmático Famatiniano de esta región, podemos sugerir que el metamorfismo $\mathrm{M} 1$ es sin a post emplazamiento de las rocas del arco, y que la fuente decalor para el evento M1, estaría vinculada con el aporte calórico proveniente del emplazamiento del magma en los niveles medios de la corteza.

En la SVF, la profundidad mínima de emplazamiento para la unidad granodiorítica de $470 \mathrm{Ma}$, fue establecida en unos $18 \mathrm{~km}$ (Murra y Baldo, 2004b). En la SLH-SI, el aumento significativo de unidades ígneas máficas y ultramáficas, sugiere que afloran niveles más profundos del arco, con unidades magmáticas emplazadas a profundidades algo mayores que las registradas en la SVF. Teniendo en cuenta lo anterior, y asumiendo una profundidad mínima de emplazamiento para las rocas ígneas de las SLH-SI entre 20 y $25 \mathrm{~km}$ (aprox. 7-8 kbar), implicaría que durante el M1, no hubo un engrosamiento cortical significativo y que las asociaciones igneas primarias (de cristalización) debieron retrogradar a las condiciones del M1.

El modelo de aproximación y posterior anexión de un terreno al borde suroccidental de Gondwana, implica que con posterioridad a la etapa de subducción y formación del arco, se debería registrar el alzamiento y descompresión de la placa litosférica superior. Esta etapa sería la registrada en la formación de las texturas de reacción coronítica del evento $\mathrm{M} 2\left(650-720^{\circ} \mathrm{C}\right.$ y 4-4,3 kbar). El alzamiento se vería favorecido por el desarrollo de fajas de cizallamiento dúctil de carácter inverso y vergencia al OSO (Murra y Baldo, 2004c). Esta actividad tectónica se habría iniciado con posterioridad a los $460 \mathrm{Ma}$, y se habría prolongado hasta los $432 \mathrm{Ma}$ (Casquet et al., 2001; Castro de Machuca et al., 2005b). 
La información geocronológica editada y discutida anteriormente, en la primera parte del trabajo, permite integrar los sectores de Chepes-Ulapes-Las Minas y SVF-SLH-SI, dentro de la misma etapa orogénica Famatiniana. Sin embargo, a partir de los trabajos de Rapela etal. (1998), Pankhurst et al. (2000) y Dahlquist y Galindo (2004), más las evidencias presentadas en este trabajo, es posible establecer que el arco magmático del Orógeno Famatiniano a los $30-32^{\circ} \mathrm{S}$, presenta una zonación trasversal, pudiéndose diferenciar tres ambientes principales:

1. Un ambiente del arco magmático oriental, emplazado enteramente sobre un basamento Pampeano y representado por plutones aislados e intrusivos filonianos de composición tonalítica, trondhjemítica y granodiorítica, con edades de 490-480 Ma (serie TTG, Pankhurst et al., 2000).

2. Un ambiente de arco magmático central, que ocupa las sierras de Los Llanos, Chepes y Las Minas-Ulapes, conla formación de unidadesmagmáticas de dimensiones batolíticas compuestas por rocas intermedias (granodioritas y tonalitas), escasos monzogranitos muscovíticos, y granitos " $S$ " de origen anatéctico. La profundidad de emplazamiento de los plutones es relativamente somera (3 a $4 \mathrm{kbar},-10$ a $12 \mathrm{~km}$ ), y su edad de cristalización es entre 483 y $479 \mathrm{Ma}$ (Dahlquist, 2000; MurrayBaldo, 2000; Pankhurst etal., 2000).

3. Un ambiente del arco magmático occidental, loca- lizado en las SVF-SLH-SI con rocas ígneas intermedias (granodioritas, tonalitas y dioritas), máficas (gabronoritas y noritas) y ultramáficas (peridotitas y piroxenitas), con escasos granitos y pegmatitas tardías, emplazados a profundidades intermedias (mínimo $20 \mathrm{~km}$ ).

Igualmente, las unidades metamórficas permiten diferenciar ambientes con gradientes geotérmicos diferentes y trayectorias P-T particulares. En las sierras de Chepes-Ulapes se registra un metamorfismo progradante de baja presión, con gradientes de campo de $50-100^{\circ} \mathrm{C} / \mathrm{km}$ (Dahlquist y Baldo, 1996; Pankhurst et al., 1998).

En las SLH-Sl, el metamorfismo es de grado medio a alto $\left(\mathrm{ca} .750^{\circ} \mathrm{C}\right)$, con presiones máximas cercanas a los $9 \mathrm{kbar}$. Hacia el norte, en la SVF, igualmente las paragénesis de $\mathrm{Crd}+\mathrm{Grt}+\mathrm{Sil}$ en migmatitas, indican un metamorfismo de alta temperatura con presiones intermedias; pero más al oeste, en el sector de Loma de Las Chacras y Pie de Palo, el evento metamórfico de 460 Ma registra un gradiente de temperatura y presión diferente, con valores de presión superiores a $10 \mathrm{kbar}$ (10 a $13 \mathrm{kbar}$, Baldo et al., 2001; Casquet et al., 2001). La zonación magmática y metamónfica que muestra el orógeno Famatiniano a los $32^{\circ} 30^{\prime}$ s, es también consistente con el modelo de aproximación de un terreno al borde continental, emplazamiento de un arco magmático sobre corteza continental, seguido de colisión y alzamiento diferencial.

\section{AGRADECIMIENTOS}

Este trabajo forma parte de la tesis doctoral del primer autor, presentada en Universidad Nacional de Córdoba, Argentina. Las tareas de campo y laboratorio fueron subsidiadas a través de los Proyectos PIP CONICET No. 02082, SECYT-UNC 2002-2003 (Argentina) y BTE 2001-1486 MCYT (España). Un especial agradecimiento al Centro de Microscopía
Electrónica Luis Bru de la Universidad Complutense de Madrid, y a la colaboración de Pablo Alasino en los trabajos de campo. Los autores agradecen a los revisores del trabajo, Dra. G. Vujovich y Dr. D. Morata por los importantes aportes realizados, y especialmente al Dr. C. Casquet por la lectura crítica y sugerencias.

\section{REFERENCIAS}

Aceñolaza, G.; Toselli, A. 1973. Consideraciones estratigráficas y tectónicas sobre el Paleozoico inferior del noroeste argentino. In Congreso Latinoamericano de Geología, No. 2, Actas 2: 755-763.
Astini, R.; Thomas, W. 1999. Origin and evolution of the Precordillera Terrane of western Argentina: a drifted Laurentian orphan. In Laurentia-Gondwana connections before Pangea (Ramos, V.; Keppie, J.; 
editores). Geological Society of America, Special Paper 336: 1-20.

Astini, R.; Ramos, V.; Benedetto, L.; Vaccari E.; Cañas, F. 1996. La Precordillera: Un terreno exótico a Gondwana. In Congreso Geológico Argentino, No. 13, 5: 273-292. Buenos Aires.

Baldo, E.; Casquet, C.; Rapela, C.; Pankhurst, R.; Galindo, C.; Fanning, M.; Saavedra, J. 2001. Ordovician metamorphism at the southwestern margin of Gondwana: P-T conditions and U-Pb SHRIMP ages from Loma de Las Chacras, Sierras Pampeanas. In South American Symposium on Isotope Geology No. 3. Abstracts. (CD-ROM). Pucón.

Berman, R. 1991. Thermobarometry using multi-equilibrium calculations: a new technique, with petrological applications. Canadian Mineralogist 29: 833-855.

Berman, R.; Aranovich, L. 1996. Optimized standard state and mixing properties of minerals: I. Model calibration for olivine, orthopyroxene, cordierite, garnet, and ilmenite in the system FeO-MgO-CaO- $\mathrm{Al}_{2} \mathrm{O}_{3}-$ $\mathrm{TiO}_{2}-\mathrm{SiO}_{2}$. Contributions to Mineralogy and Petrology 126 (1-2): 1-24.

Bucher, K.; Frey, M. 1994. Petrogenesis of metamorphic rocks. Springer-Verlag Berlin Heidelberg. Edition No. 6: $318 \mathrm{p}$.

Caminos, R. 1979. Descripción geológica de las hojas 21f, sierra de las Minas y $21 \mathrm{~g}$, Ulapes. Servicio Geológico Nacional. Boletin 172 (Inédito). Buenos Aires

Casquet, C.; Baldo, E.; Pankhurst, R.; Rapela, C.; Galindo, C.; Fanning, M.; Saavedra, J. 2001. Involvement of the Argentine Precordillera terrane in the Famatinian mobile belt: U-Pb SHRIMP and metamorphic evidence from the Sierra de Pie de Palo. Geology 29 (8): 703706.

Casquet, C.; Galindo, C.; Rapela, C.; Pankhurst, R.; Baldo, E.; Saavedra, J.; Dahlquist, J. 2003. Granate con alto contenido en tierras raras pesadas (HREE) y elevada relación $\mathrm{Sm} / \mathrm{Nd}$, en pegmatitas de la sierra de Valle Fértil (Sierras Pampeanas, Argentina). In Congreso de Mineralogía y Petrologia SEM/03 (Sociedad Española de Mineralogía) No. 23. Publicación del Boletin de la Sociedad Española de Mineralogia 25-A: 133-134.

Castro de Machuca, B.; Conte-Grand, A.; MeissI, E.; Pontoriero, S.; Sumay, C. 1996. Petrología de las asociaciones máficas-ultramáficas de la Sierra de La Huerta, San Juan, Argentina. In Congreso Geológico Argentino No. 13 y Congreso de Explotación de Hidrocarburos No. 3, 3: 439-452. Buenos Aires.

Castro de Machuca, B.; Conte-Grand, A.; Meissl, E.; Pontoriero, S.; Recio, G.; Sumay, C. 2002. Mineralogy and textures of metagabbros and ultramafic related rocks from La Huerta and Valle Fertil ranges, western Pampean Ranges, San Juan, Argentina. InMineralogía y Metalogenia (Brodtkorb, M.; Koukharsky, M.; Leal, R.; editores). Congreso de Mineralogia y Metalogenia, No. 6: 67-75. Buenos Aires.
Castro de Machuca, B.; Morata, D.; Arancibia, G.; Belmar, M.; Pontoriero, S. 2004. Metamorphic evolution of high-grade metagabbros from La Huerta Range, western Pampean Ranges, San Juan, Argentina. International Geological Congress, No. 32. CD-ROM Edition. Florence.

Castro de Machuca, B.; Arancibia, G.; Morata, D.; Belmar, M.; Pontoriero, S.; Previley, L. 2005a. Transformaciones texturales, mineralógicas y químicas en metagabros afectados por cizallamiento dúctil, Sierra de La Huerta, San Juan, Argentina. In Congreso Geológico Argentino, No. 14, Actas (CD-ROM). La Plata.

Castro de Machuca, B.; Arancibia, G.; Morata, D.; Beldar, M.; Pontoriero, S. 2005b. Early Silurian medium-grade shear zone in the La Huerta range, Western Pampean Ranges, Argentina: implications for Gondwana margin evolution. In Gondwana No. 12: Geological and biological heritage of Gonwana (Pankhurst, R.; Veiga, G.; editores). Academia Nacional de Ciencias, Abstracts, 93.

Dahlquist, J. 2000. Geologia, petrologia y geoquimica de las rocas ígneas y metamórficas de las Sierras de Chepes, La Rioja, Argentina. Tesis Doctoral (inédito). Facultad de Ciencias Exactas, Fisicas y Naturales, Universidad Nacional de Córdoba: 464 p.

Dahlquist, J.; Baldo, E., 1996. Metamorfismo y deformación famatinianos en la Sierra de Chepes. In Congreso Geológico Argentino No. 13, Simposio Internacional Proto-Andean Margin of Gondwana, 5: 393-409. Buenos Aires.

Dahlquist, J.; Galindo, C. 2004. Geoquímica isotópica de los granitoides de la Sierra de Chepes: un modelo geotectónico y termal, implicancias para el orógeno famatiniano. Revista de la Asociación Geológica Argentina 59 (1): 57-69.

Dahlquist, J.; Rapela, C.; Baldo, E. 2005. Petrogenesis of cordierite-bearing S-Type granitoids in the Sierra de Chepes, Famatinian Orogen, Argentina. Journal of South American Earth Science 20: 231-251.

Ellis, D. 1980. Osumilite-sapphirine-quartz granulites from Enderby Land, Antartica: P-T conditions of metamorphism, implications for garnet-cordierite equilibria and the evolution of the deep crust. Contributions to Mineralogy and Petrology 74: 201210.

Fuhrman, M.; Lindsley, D. 1988. Ternary-feldspar modeling and thermometry. American Mineralogist 73: 201-216.

Galindo, C.; Casquet, C.; Rapela, C.; Pankhurst, R.; Baldo, E.; Saavedra, J. 2004a. Sr, Cand O isotope geochemistry and stratigraphy of Precambrian and lower Paleozoic carbonate sequences from the Western Sierras Pampeanas of Argentina: tectonic implications. Precambrian Research 131 (1-2): 55-71.

Galindo, C.; Murra, J.; Baldo, E.; Casquet, C.; Rapela, C.; Pankhurst, R.; Dahlquist, J. 2004b. Datación Sm-Nd 
del metamorfismo en la Sierra de las Imanas (Sierras Pampeanas Occidentales, Argentina). Geogaceta 35: $75-78$

Gasparik, T.; Newton, R. 1984. The reversed alumina contents of orthopyroxene in equilibrium with spinel and forsterite in the system $\mathrm{MgO}-\mathrm{Al}_{2} \mathrm{O}_{3}-\mathrm{SiO}_{3}$. Contributions to Mineralogy and Petrology 85: 186196.

Graessner, T.; Schenk, V. 2001. An exposed Hercynian deep crustal section in the Sila Massif of northern Calabria: path of granulite-facies metapelitic migmatites and metabasites. Journal of Petrology 5 (42): 931 961.

Graham, C.; Powell, R. 1984. A garnet-hornblende geothermometer: calibration, testing and application to the Pelona Schist, Southern California. Journal of Metamorphic Geology 2: 13-31.

Holland, T.; Blundy, J. 1994. Non-ideal interactions in calcic amphiboles and their bearing on amphiboleplagioclase thermometry. Contributions to Mineralogy and Petrology 116: 433-447.

Hollister, L.; Grissom, G.; Peters, E.; Stowell, H.; Sisson, V. 1987. Confirmation of empirical correlation of $\mathrm{Al}$ in hornblende with pressure of solidification of calcalkaline plutons. American Mineralogist 72: 231-239.

Jarosewich, E.; Nelen, J.; Norberg, J. 1980. Reference samples for electron microprobe analysis. Geostandards Newsletter 4: 43-47.

Jordan, T.; Allmendinger, R. 1986. The Sierras Pampeanas of Argentina: a modern analogue of Rocky Mountain foreland deformation. American Journal of Science 286: 737-764.

Kohn, M.; Spear, F. 1989. Empirical calibration of geobarometers for the assemblage garnet+ hornblende +plagioclase+quartz. American Mineralogist 74: 77-84.

Kretz, R. 1983. Symbols for rock-forming minerals. American Mineralogist 68: 277-279.

Leake, B.; Woolley, A.; Arps, C.; Birch, W.; Gilbert, M.; Grice, J.; Hawthorne, F.; Kato, A.; Kisch, H.; Krivovichev, V.; Linthout, K.; Laird, J.; Mandarino, J.; Maresch, W.; Nickel, E.; Rock, N.; Schumacher, J.; Smith, D.; Stephenson, N.; Ungaretti, L.; Whittaker, E.; Youzhi, G. 1997. Nomenclature of amphiboles. Report of Subcomittee on Amphiboles of the International Mineralogical Association Commission on New Minerals and Mineral Names. European Journal of Mineralogy 9: 103-119.

Mader, U.; Berman R. 1992. Amphibole thermobarometry: a thermodynamic approach. Current Research, Part E, Geological Survey of Canada Paper 92-1E: 393400

McDonough, M.; Ramos, V.; Isachsen, C.; Bowring, S.; Vujovich, G. 1993. Nuevas edades de circones del basamento de la sierra de Pie de Palo, Sierras Pampeanas occidentales de San Juan: sus implicancias para los modelos del supercontinente proterozoico de Rodinia. In Congreso Geológico Argentino No. 12 y Congreso de Exploración de Hidrocarburos No. 2, 3: 340-342. Mendoza.

Murra, J. 2004. Estudio petrológico-estructural del borde occidental del orógeno Famatiniano (sierra de Valle Fértil-La Huerta), y su comparación con el sector central (sierra de Las Minas-Ulapes), provincias de San Juan y La Rioja. Tesis Doctoral (inédito). Facultad de Ciencias Exactas, Fisicas y Naturales, Universidad Nacional de Córdoba. $329 \mathrm{p}$.

Murra, J.; Baldo, E. 2000. Condiciones de cristalización del magmatismo Famatiniano en las Sierras de Las Minas-Ulapes Sierras Pampeanas: correlación con otros sectores. In Congreso Geológico Chileno No. 9. Actas, Resúmenes Expandidos 1: 659-663. Puerto Varas.

Murra, J.; Baldo, E. 2001. Metamorfismo y deformación en La Sierra de las Imanas, margen occidental del orógeno Famatiniano, Sierras Pampeanas Argentinas. In Congreso Geológico Latinoamericano No. 11, Abstracts, 3: 18. Montevideo.

Murra, J.; Baldo, E. 2004a. Texturas coroníticas en las rocas ultramáficas de las sierras de La Huerta y de Las Imanas: caracterización y estimaciones P-T. In Avances en Mineralogia, Metalogenia y Petrologia: 373378.

Murra, J.; Baldo, E. 2004b. Condición de emplazamiento de la granodiorita Valle Fértil y su comparación con las granodioritas del batolito los Llanos-Ulapes. In Avances en Mineralogia, Metalogenia y Petrología 2004: 367-372.

Murra, J.; Baldo, E. 2004c. Las fajas de cizalla dúctil en la sierra de Las Imanas, Provincia de San Juan, Argentina. In Reunión sobre Microtectónica y Geología Estructural No. 12. Resümenes: 26. Cafayate.

Pankhurst, R.; Rapela, C. 1998. The Proto-Andean margin of Gondwana: an introduction. In Proto-Andean Margin of Gondwana (Pankhurst, R.; Rapela, C. editores). Geological Society of London, Special Publication 142: $1-10$.

Pankhurst, R.; Rapela, C.; Fanning, M. 2000. Age and origin of coeval TTG, I- and S-type granites in the Famatinian belt of NW Argentina. Transactions of Royal Society of Edinburgh. Earth Sciences 91: 151 168.

Pankhurst, R.; Rapela, C.; Saavedra, J.; Baldo, E.; Dahlquist, J.; Pascua, I. 1998. The Famatinian magmatic arc in the central of Sierras Pampeanas. In Proto-Andean Margin of Gondwana (Pankhurst, R.; Rapela, C. editores). Geological Society of London, Special Publication 142: 343-368.

Powell, R.; Holland, T. 1988. An internally consistent dataset with uncertainties and correlations: Applications to geobarometry, worked examples and a computer program. In Journal of Metamorphic Geology 6: 173-204.

Ramos, V. 1999. Geologia Argentina. Servicio Geológico 
Minero Argentino, Anales 29 (3): 41-96. Buenos Aires.

Ramos, V.; Jordan, T.; Allmendinger, R.; Mpodozis, C.; Kay, S.; Cortes, S.; Palma, J. 1996. Paleozoic terranes of the central Argentine-Chilean Andes. Tectonics 5: 855-880.

Rapela, C.; Pankhurst, R.; Casquet, C.; Baldo, E.; Saavedra, J.; Galindo, C.; Fanning, C. 1998. The Pampean orogen of the Southern proto-Andean Cambrian continental collision in the Sierras de Cordoba. In The proto-Andean Margin of Gondwana (Pankhurst, R.; Rapela, C.; editores). Geological Society of London, Special Publication 142: 181-217.

Rapela, C.; Pankhurst, R.; Baldo, E.; Casquet, C.; Galindo, C.; Fanning, C.; Saavedra, J. 2001. Ordovician Metamorphism in the Sierras Pampeanas: New U-Pb SHRIMP ages in central-east Valle Fértil and Velasco Batholith. In South American Symposium on Isotope Geology No. 3, Extended Abstract (CD-ROM): 616 619. Pucón.

Roeder, P.; Campbell, J.; Jamieson, H. 1979. A re-evaluation of the olivine-spinel geothermometer. Contributions to Mineralogy and Petrology 68: 325-334.
Schmädicke, E. 2000. Phase relations in peridotitic and pyroxenitic rocks in the model system CMASH and CNMASH. Journal of Petrology 44: 69-86.

Sims, J.; Ireland, T.; Camacho, A.; Lyons, P.; Pieters, P.; Skirrow, R.; Stuart-Smith, P.; Miró, R. 1998. U-Pb, Th$\mathrm{Pb}$ and Ar-Ar geochronology from the southern Sierras Pampeanas, Argentina: implications for the Paleozoic tectonic evolution of the western Gondwana margin. In The proto-Andean Margin of Gondwana (Pankhurst, R.; Rapela, C. editores). Geological Society of London. Special Publication 142: 259-282.

Spear, F.; Kohn, M.; Cheney, J. 1999. P-T paths from anatectic pelites. Contributions to Mineralogy and Petrology 134: 17-32.

Vujovich, G.; Godeas, M.; Marín, G.; Pezzutti, N. 1996. El complejo magmático de la sierra de La Huerta, Prov. de San Juan. Actas. In Congreso Geológico Argentino No. 13 y Congreso de Explotación de Hidrocarburos No. 3, 3: 465-475. Buenos Aires.

Wood, B.; Banno, S. 1973. Garnet-orthopyroxene and orthopyroxene-clinopyroxene relationships in simple and complex systems. Contributions to Mineralogy and Petrology 42: 109-124. 